\title{
The Rot-Div System in Exterior Domains
}

\author{
Piotr B. Mucha and Milan Pokorný \\ Communicated by K. Pileckas
}

\begin{abstract}
The goal of this paper is to reconsider the classical elliptic system $\operatorname{rot} \boldsymbol{v}=\boldsymbol{f}, \operatorname{div} \boldsymbol{v}=g$ in simply connected domains with bounded connected boundaries (bounded and exterior sets). The main result shows solvability of the problem in the maximal regularity regime in the $L_{p}$-framework taking into account the optimal/minimal requirements on the smoothness of the boundary. A generalization for the Besov spaces is studied, too, for $\boldsymbol{f} \in \dot{B}_{p, q}^{s}(\Omega)$ for $-1+\frac{1}{p}<s<\frac{1}{p}$. As a limit case we prove the result for $\boldsymbol{f} \in \dot{B}_{3,1}^{0}(\Omega)$, provided the boundary is merely in $B_{3,1}^{2-1 / 3}$. The dimension three is distinguished due to the physical interpretation of the system. In other words we revised and extended the classical results of Friedrichs (Commun Pure Appl Math 8:551-590, 1955) and Solonnikov (Zap Nauch Sem LOMI 21:112-158, 1971).
\end{abstract}

Mathematics Subject Classification. Primary 35F35; Secondary 35J56, 35B45.

Keywords. Rot-div system, optimal regularity, exterior domain, Besov spaces, a-priori estimates.

\section{Introduction}

The subject of the present paper is to analyze solutions to the following problem

$$
\begin{aligned}
& \operatorname{rot} \boldsymbol{v}=\boldsymbol{f} \text { in } \Omega, \\
& \operatorname{div} \boldsymbol{v}=g \text { in } \Omega, \\
& \vec{n} \cdot \boldsymbol{v}=b \text { on } \partial \Omega
\end{aligned}
$$

in a three dimensional domain $\Omega$, where $\boldsymbol{v}$ is a sought vector field, $\boldsymbol{f}, g, b$ are given data and rot, div are the vorticity and divergence operators, $\vec{n}$ is the outer normal to $\partial \Omega$. Due to questions concerning the kernel of operator (1.1) we restrict ourself to simply connected domains with bounded connected boundaries. Thus we obtain two classes: bounded and exterior domains. We assume that all functions have a certain decay at infinity expressed by the corresponding spaces the functions belong to. Note that the vorticity operator is sometimes denoted by curl, however, we prefer the notation rot.

The above problem is one of the most fundamental linear systems in physics, we find it in electromagnetism (the Maxwell equations) or in fluid mechanics [14-18]. In case of incompressible fluids ( $\operatorname{div} \boldsymbol{v}=0)$ it enables to recover the velocity from the knowledge of the vorticity. In case of electromagnetism, having electric field, we are able to recover information about the magnetic field and vice-versa. It is closely related to the Helmholtz decomposition of vector fields [14]. Its two dimensional version leads to a trivial system being just a scalar Dirichlet problem for the Laplace operator. More than the three dimensional versions have no reasonable physical meaning. Thus we concentrate here our attention on the most interesting case, i.e. on the three dimensional one. From the point of view of the theory of PDEs, equations (1.1) are a nontrivial version of elliptic system of order one. Additionally, the exterior domain gets us closer to issues arising from application problems like description of flows past an obstacle.

System (1.1) can be simplified. We solve the following Neumann problem

$$
\begin{aligned}
& \Delta p=g \text { in } \Omega, \\
& \frac{\partial p}{\partial \vec{n}}=b \text { on } \partial \Omega .
\end{aligned}
$$


We are required to assume the following compatibility condition $\int_{\Omega} g \mathrm{~d} x=\int_{\partial \Omega} b \mathrm{~d} \sigma$ at least in a weak sense, provided the integrals exist. Subtracting the gradient of a solution to (1.2) from a solution to (1.1), and substituting

$$
\boldsymbol{v} \leftrightarrow \boldsymbol{v}-\nabla p \text { with } \boldsymbol{f} \leftrightarrow \boldsymbol{f}-\operatorname{rot} \nabla p=\boldsymbol{f},
$$

we arrive in (1.1) to the following canonical form

$$
\begin{aligned}
& \operatorname{rot} \boldsymbol{v}=\boldsymbol{f} \text { in } \Omega, \\
& \operatorname{div} \boldsymbol{v}=0 \text { in } \Omega, \\
& \vec{n} \cdot \boldsymbol{v}=0 \text { on } \partial \Omega .
\end{aligned}
$$

The compatibility condition to system (1.4) says that

$$
\operatorname{div} \boldsymbol{f}=0,
$$

since div rot $\equiv 0$.

The main objective of the present paper is the issue of existence and uniqueness to system (1.4). We consider our problem in the setting of Sobolev-Besov spaces. The first result reads

Theorem 1.1. Let $\Omega \subset \mathbb{R}^{3}$ be a simply connected domain with a connected bounded boundary $\partial \Omega \in W_{q}^{2-1 / q}$, $1<p<\infty$, where $q=p$ if $p>3$ and $q>3$, arbitrary, if $p \leq 3$. Let $\boldsymbol{f} \in L_{p}\left(\Omega ; \mathbb{R}^{3}\right)$ with $\operatorname{div} \boldsymbol{f}=0$ in $\Omega$ and $\boldsymbol{f} \cdot \vec{n}=0$ at $\partial \Omega$.

Then there exists unique solution $\boldsymbol{v}$ to (1.4) with $\nabla \boldsymbol{v} \in L_{p}\left(\Omega ; \mathbb{R}^{3 \times 3}\right)$ and

$$
\|\nabla \boldsymbol{v}\|_{L_{p}(\Omega)} \leq C\|\boldsymbol{f}\|_{L_{p}(\Omega)} .
$$

Remark 1.2. The uniqueness of solutions to (1.4) for domains with $\partial \Omega$ bounded, connected is easy to show. Let $\boldsymbol{v} \in L_{1, l o c}\left(\Omega ; \mathbb{R}^{3}\right)$ with $\nabla \boldsymbol{v} \in L_{p}\left(\Omega ; \mathbb{R}^{3 \times 3}\right)$ solve in the weak sense the following problem

$$
\begin{aligned}
\operatorname{rot} \boldsymbol{v} & =\mathbf{0} \text { in } \quad \Omega, \\
\operatorname{div} \boldsymbol{v} & =0 \text { in } \Omega, \\
\vec{n} \cdot \boldsymbol{v} & =0 \text { on } \partial \Omega .
\end{aligned}
$$

We claim that $\boldsymbol{v}=\mathbf{0}$. Since the first group of homology $\pi_{1}(\Omega)$ is trivial - the domain is simply connected, so the Poincaré lemma (see e.g. [20]) says (we use the fact that $\partial \Omega$ is connected and bounded and $\Omega \subset \mathbb{R}^{3}$ ) that if $\operatorname{rot} \boldsymbol{v}=0$ then there exists a scalar function $h$ with $\nabla h \in W_{p}^{1}\left(\Omega ; \mathbb{R}^{3}\right)$ such that $\boldsymbol{v}=\nabla h$. Then (1.6) reads

$$
\begin{aligned}
\Delta h & =0 \text { in } \Omega, \\
\vec{n} \cdot \nabla h & =0 \text { on } \partial \Omega,
\end{aligned}
$$

so the maximum principle yields $\nabla h=\mathbf{0}$, i.e. $\boldsymbol{v} \equiv \mathbf{0}$. Similar arguments yield the uniqueness also in the subsequent theorems.

The next result generalizes Theorem 1.1 on the Besov spaces.

Theorem 1.3. Let $\Omega \subset \mathbb{R}^{3}$ be a simply connected domain with a connected bounded boundary $\partial \Omega \in$ $B_{r, q}^{2+s-1 / r}, 1<p<\infty, q \in[1, \infty],-1+\frac{1}{p}<s<\frac{1}{p},-1+\frac{3}{r}<s$, where $r=p$ if $p>3$ and $r>3$, arbitrary, if $p \leq 3$. Let $\boldsymbol{f} \in \dot{B}_{p, q}^{s}\left(\Omega ; \mathbb{R}^{3}\right)$ with $\operatorname{div} \boldsymbol{f}=0$ in $\Omega$ and $\boldsymbol{f} \cdot \vec{n}=0$ at $\partial \Omega$.

Then there exists unique solution $\boldsymbol{v}$ to $(1.4)$ with $\nabla \boldsymbol{v} \in \dot{B}_{p, q}^{s}\left(\Omega ; \mathbb{R}^{3 \times 3}\right)$ and

$$
\|\nabla \boldsymbol{v}\|_{\dot{B}_{p, q}^{s}(\Omega)} \leq C\|\boldsymbol{f}\|_{\dot{B}_{p, q}^{s}(\Omega)} .
$$

Note that the restriction $s<\frac{1}{p}$ is connected with the fact that we want to avoid the necessity to consider additional compatibility conditions which would naturally appear for $s$ large. 
The limit case of the above theorem, for the optimal regularity of the boundary in the critical Besov spaces, is the following.

Theorem 1.4. Let $\Omega \subset \mathbb{R}^{3}$ be a simply connected domain with a connected bounded boundary $\partial \Omega \in B_{3,1}^{2-\frac{1}{3}}$ and $\boldsymbol{f} \in \dot{B}_{3,1}^{0}\left(\Omega ; \mathbb{R}^{3}\right)$ with $\operatorname{div} \boldsymbol{f}=0$ in $\Omega$ and $\boldsymbol{f} \cdot \vec{n}=0$ at $\partial \Omega$.

Then there exists unique solution $\boldsymbol{v}$ to $(1.4)$ with $\nabla \boldsymbol{v} \in \dot{B}_{3,1}^{0}\left(\Omega ; \mathbb{R}^{3 \times 3}\right)$ and

$$
\|\nabla \boldsymbol{v}\|_{\dot{B}_{3,1}^{0}(\Omega)} \leq C\|\boldsymbol{f}\|_{\dot{B}_{3,1}^{0}(\Omega)} .
$$

Note that in situations considered in Theorems 1.3 and 1.4 the normal component of the trace of $\boldsymbol{f}$ is well defined, see [8]. The assumption put on the domain implies that we consider only bounded sets being diffeomorphic with a ball or exterior domains such that $\mathbb{R}^{3} \backslash \Omega$ is a set diffeomorphic with a ball. Such restriction guarantees the uniqueness of considered problems in the proofs. Throughout the whole paper we consider only domains with boundary regularity at least $C^{1,0}$.

To describe the mathematical motivation of our paper, we first note that this important system has not been studied so intensively in the mathematical literature. The classical results are due to Friedrichs [13] and Solonnikov [26], some extensions of these results one can find in [2,9,24,31]. Note that some of the current results for this system concern numerical analysis [9,24]. The papers [1] and [30] study the problem in the $L^{p}$ framework ([1] also in the $W^{k, p}$ framework) also in domains with non-zero Betti's number. However, the authors assume that the boundary is sufficiently smooth. They also considered several types of boundary conditions. In [6], the result was extended to Hölder spaces. All three papers were based on estimates of corresponding potentials.

Here we want to reconsider the proof, giving some extension for solvability in the Besov class. Our method is based on the a priori bounds and compactness, omitting technical theory of potentials. Additionally, we examine rigorously the case of exterior domain. As the main new result we obtain the existence in the limit Besov spaces with the lowest regularity of the boundary (see Theorem 1.4), such results are necessary in order to consider free boundary problem in different areas of fluid mechanics and electromagnetism.

Let us discuss the chosen regularity. We consider regular solutions, i.e., the gradient of the solution is integrable, or at least almost integrable. We shall look closer at the choice of regularity of the boundary which is a key element of our considerations. In Theorem 1.1 it is required that $\partial \Omega \in W_{q}^{2-1 / q}$ with $q>3$. It follows that the normal vector field is at least Hölder continuous $C^{0, \alpha}$ with $\alpha=1-3 / p$. Next, Theorem 1.3 extends the results for Sobolev spaces on the Besov setting, we are interested only in the case as $s$ is in a neighborhood of zero, since such considerations are related to the issue of optimal regularity. One can find this restriction in current works concerning free boundary problems in the optimal regularity [10-12,21-23,27,28,32]. Theorem 1.4 reaches the limit case, namely, $\partial \Omega \in B_{3,1}^{5 / 3}$, what implies that the normal vector field is $C^{\omega(\cdot)}$ with some modulus of continuity $\omega(\cdot)$.

The paper is organized as follows. First we reformulate the problem into the second order elliptic equations and present the necessary definitions and few elementary facts for the Laplace operator and for products in Besov spaces. In Sect. 3 we prove the elementary existence of $L_{2}$ solutions taking care of the regularity of the boundary. Next, we consider the $L_{p}$ case, showing that the weak solution can be of better regularity. Hence we obtain the result in the Sobolev spaces. In Remark 4.4 we explain why the proof of Theorem 1.3 is not given. See also Remark 3.5. At the end we investigate the limit case, i.e., we prove Theorem 1.4.

\section{Preliminaries}

An effective approach to system (1.4) is via reduction to a second order elliptic problem. Note that we use the result below in Sect. 3 in order to construct solutions to our problem for the right-hand side $f$ sufficiently smooth (in fact, square integrable) and with compact support. Therefore we get solutions 
which are in fact regular (in $W_{2}^{2}\left(\Omega ; \mathbb{R}^{3}\right)$ ) and the following result shows the solutions to (2.1) below are closely connected to the solutions of our main system (1.4).

Proposition 2.1. Let $\boldsymbol{f} \in L_{2}\left(\Omega ; \mathbb{R}^{3}\right)$ with $\operatorname{div} \boldsymbol{f}=0$. Let $\boldsymbol{e} \in W_{2}^{2}\left(\Omega ; \mathbb{R}^{3}\right)$ be a solution to the following system $^{1}$

Then

$$
\begin{aligned}
& -\Delta \boldsymbol{e}=\boldsymbol{f} \text { in } \Omega, \\
& \boldsymbol{e} \cdot \vec{\tau}_{k}=0 \text { at } \partial \Omega \quad k=1,2, \quad \int_{\partial \Omega} \boldsymbol{e} \cdot \vec{n} \mathrm{~d} \sigma=0 . \\
& \operatorname{div} \boldsymbol{e}=0 \text { at } \partial \Omega,
\end{aligned}
$$

$$
\boldsymbol{v}:=\operatorname{rot} \boldsymbol{e} \in W_{2}^{1}\left(\Omega ; \mathbb{R}^{3}\right)
$$

is a solution to (1.4).

Proof. We will show that a solution to (2.1) generates a solution to (1.4). Taking $\boldsymbol{v}=\operatorname{rot} \boldsymbol{e}$ we immediately get $(1.4)_{1,2}$, because $\Delta \boldsymbol{e}=-\operatorname{rot} \operatorname{rot} \boldsymbol{e}=-\operatorname{rot} \boldsymbol{v}$ and div rot $\equiv 0$. To obtain $(1.4)_{3}$ note that $\vec{n} \cdot \operatorname{rot} \boldsymbol{e}=0$ at $\partial \Omega$, since without loss of generality we may take $\vec{n}=(1,0,0)$, then $\vec{n} \cdot \operatorname{rot} \boldsymbol{e}=0$, because $e_{, 2}^{3}-e_{, 3}^{2}=0-0=0$. Here we used that $e^{2}, e^{3}$ are zero as tangent parts of $\boldsymbol{e}$, so their tangent derivatives are zero at this point, too.

Remark 2.2. Indeed, condition $\int_{\partial \Omega} \boldsymbol{e} \cdot \vec{n} \mathrm{~d} \sigma=0$ is irrelevant in order to show that solutions to (2.1) generate solutions to (1.4). On the other hand, this condition allows us to get unique solutions to (2.1) which is useful in order to get estimates of solutions to (2.1) in different spaces and thus construct solutions to our main problem.

Note that the condition $\int_{\partial \Omega} e \cdot \vec{n} \mathrm{~d} \sigma=0$ for a solution to (2.1) is automatically fulfilled in the case of a bounded domain with connected boundary; it is a consequence of the global constraint div $\boldsymbol{e}=0$ coming from the fact that div $\boldsymbol{f}=0$, and the boundary condition $(2.1)_{3}$. But in the case of exterior problem or in the case when the boundary consists of several connected parts we are able to obtain a nontrivial kernel of the operator without this integral constraint. Since $\partial \Omega$ is connected in our case this condition is sufficient, but in the general case, a suitable modification of this constraint is required. As an example it is enough to consider the system

$$
\Delta h=0,\left.\quad h\right|_{\partial \Omega}=1 \text { and } h \rightarrow 0 \text { as }|x| \rightarrow \infty,
$$

with $\Omega=\mathbb{R}^{3} \backslash \overline{B_{1}(0)}$. Hence $h=\frac{1}{|x|}$; taking $\boldsymbol{e}=\nabla h$ system (2.1) is fulfilled (with $\boldsymbol{f} \equiv \mathbf{0}$ ), but of course $\int_{\partial \Omega} \boldsymbol{e} \cdot \vec{n} \mathrm{~d} \sigma=0$ does not hold.

Hence we reduced our consideration to analysis of the well posedness of system (2.1).

To analyze precisely regularity of solutions in a domain $\Omega$ we are required to construct a suitable localization. Given $\lambda>0$, a parameter which will be specified later (and may depend on the point at the boundary), we choose a compact set $K$ such that

$$
K \subset \bar{\Omega}, \quad \operatorname{dist}\{\partial K \backslash \partial \Omega, \partial \Omega\} \geq \lambda \text { and } \lambda \leq \operatorname{dist}\{\Omega \backslash K, \partial \Omega\} \leq 2 \lambda .
$$

Then we introduce a smooth function $\eta^{0}: \mathbb{R}^{3} \rightarrow[0,1]$ such that

$$
\eta^{0}=\left\{\begin{array}{llrl}
1 & \text { for } & x: \operatorname{dist}\{x, \partial \Omega\} & >\frac{\lambda}{4} \\
\in[0,1] & \text { for } & x & \in K \\
0 & \text { for } & x & \in \partial \Omega
\end{array}\right.
$$

and supp $\nabla \eta^{0} \subset \subset$ int $K$, in particular supp $\nabla^{0} \eta \cap \partial \Omega=\emptyset$.

The second step are considerations near the boundary. Given $x_{0} \in \partial \Omega$ we find $\eta^{x_{0}}: \mathbb{R}^{3} \rightarrow[0,1]$ such that

then $\operatorname{supp} \eta^{x_{0}} \cap \Omega \subset K$.

$$
\eta^{x_{0}}(x)=\left\{\begin{array}{llr}
1 & \text { for } & x \in B\left(x_{0}, \frac{\lambda}{2}\right) \\
\in[0,1] & \text { for } r & x \in K \\
0 & \text { for } x & \mathbb{R}^{3} \backslash B\left(x_{0}, \lambda\right)
\end{array}\right.
$$

\footnotetext{
${ }^{1} \vec{\tau}_{k}$ denotes the tangent vector to $\partial \Omega$.
} 
We see that

$$
\left\{x \in \Omega: \operatorname{dist}\{x, \partial \Omega\}>\frac{\lambda}{4}\right\} \cup\left(\bigcup_{y \in \partial \Omega} B(y, \lambda / 2) \cap \Omega\right)=\Omega .
$$

Moreover, for fixed $\lambda>0$ there exists a finite subcovering, i.e. $\left\{x_{k}\right\}_{k \in I}$ with $I=\left\{1, \ldots, N_{\lambda}\right\}$ such that (recall that $\partial \Omega$ is bounded)

$$
\left\{x \in \Omega: \operatorname{dist}\{x, \partial \Omega\}>\frac{\lambda}{4}\right\} \cup\left(\bigcup_{k \in I} B\left(x_{k}, \lambda / 2\right) \cap \Omega\right)=\Omega .
$$

Consider the case $\partial \Omega \in C^{2}$. For each $x_{0} \in \partial \Omega$ we find a map

$$
Z_{x_{0}}: \mathbb{R}_{+}^{3} \cap B(0, \lambda) \rightarrow \Omega \cap B\left(x_{0}, \lambda\right)
$$

such that $Z(0)=x_{0}$ and $d Z(0)$ is such that $d Z(0)\left(\partial \mathbb{R}_{+}^{3}\right)=T_{x_{0}} \partial \Omega$, in addition $Z_{x_{0}} \in C^{2}$, too, and for all $z \in \mathbb{R}_{+}^{3} \cap B(0, \lambda)$ there holds $|d Z(z)-d Z(0)| \leq C \lambda$.

In case $\partial \Omega \in W_{p}^{2-1 / p}$ only, we have that $Z_{x_{0}} \in W_{p}^{2}\left(\mathbb{R}_{+}^{3} \cap B(0, \lambda)\right)$ and $|d Z(z)-d Z(0)| \leq C \lambda^{\alpha}$ with $\alpha=1-3 / p$. Finally, in the case of the limit regularity, namely $\partial \Omega \in B_{3,1}^{5 / 3}$, there exists a modulus of continuity $\omega(\cdot)$ and then $|d Z(z)-d Z(0)| \leq \omega(|z-0|)$.

Next we introduce the basic function spaces $[3,4,29]$. By $L_{p}(\Omega)$ we denote the usual Lebesgue space of functions integrable with the $p$-th power over the domain $\Omega$. The Sobolev space $W_{p}^{k}(\Omega)$ is endowed with the following norm

$$
\|f\|_{W_{p}^{k}(\Omega)}=\|f\|_{L_{p}(\Omega)}+\sum_{0<|\alpha| \leq k}\left\|\partial^{\beta} f\right\|_{L_{p}(\Omega)},
$$

where $\beta=\left(\beta_{1}, \ldots, \beta_{k}\right) \in \mathbb{N}^{k}$ stands for multi-index and $|\beta|=\beta_{1}+\cdots+\beta_{k}$ as well as $\partial^{\beta}=\partial_{x_{1}}^{\beta_{1}} \ldots \partial_{x_{k}}^{\beta_{k}}$.

Let us recall the definition of the Besov spaces $B_{p, q}^{s}$ on $\mathbb{R}^{n}$. We do it just for $s \in(0,1]$, since for $s>1$, if $f \in B_{p, q}^{s}$, then $\nabla f \in B_{p, q}^{s-1}$ and for positive index $s$ one can define the space by induction as follows

$$
\|f\|_{B_{p, q}^{s}\left(\mathbb{R}^{n}\right)} \simeq\|f\|_{B_{p, q}^{s-1}\left(\mathbb{R}^{n}\right)}+\|\nabla f\|_{B_{p, q}^{s-1}\left(\mathbb{R}^{n}\right)} \text { for } s>1 .
$$

Let

$$
\Delta_{h} f(x)=f(x+h)-f(x), \quad x, h \in \mathbb{R}^{n} ;
$$

the modulus of continuity is defined by

$$
\omega_{p}^{2}(f, t)=\sup _{|h| \leq t}\left\|\Delta_{h}^{2} f\right\|_{L^{p}\left(\mathbb{R}^{n}\right)} .
$$

Just to point the connection to the standard modulus of continuity we note that

$$
\omega(t)=\omega(f, t)=\omega_{\infty}^{1}(f, t)=\sup _{|h| \leq t}\left\|\Delta_{h} f\right\|_{L^{\infty}\left(\mathbb{R}^{n}\right)} .
$$

Let $s \in(0,1]$. The Besov space $B_{p, q}^{s}\left(\mathbb{R}^{n}\right)$ contains all functions $f$ such that $f \in L_{p}\left(\mathbb{R}^{n}\right)$ and $\int_{0}^{\infty}\left|\frac{\omega_{p}^{2}(f, t)}{t^{s}}\right|^{q} \frac{d t}{t}$ $<\infty$. It is endowed with the norm

$$
\|f\|_{B_{p, q}^{s}\left(\mathbb{R}^{n}\right)}=\left(\|f\|_{L_{p}\left(\mathbb{R}^{n}\right)}^{q}+\int_{0}^{\infty}\left|\frac{\omega_{p}^{2}(f, t)}{t^{s}}\right|^{q} \frac{\mathrm{d} t}{t}\right)^{1 / q} .
$$

Let us note that in general $B_{p, q}^{1}\left(\mathbb{R}^{n}\right) \neq W_{p}^{1}\left(\mathbb{R}^{n}\right)$, more precisely, for $p \neq 2$ we can not find $q$ to fulfill the identity. Moreover, we used $\Delta_{h}^{2}$, instead of single $\Delta_{h}$, to cover the limit case $s=1$.

Next, we recall that for any domain $\Omega$ with sufficiently smooth boundary (Lipschitz continuity is enough), the Besov space $B_{p, q}^{s}(\Omega)$ stands for the restriction (in the distributional meaning) of functions 
in $B_{p, q}^{s}\left(\mathbb{R}^{n}\right)$ to $\Omega$. That is $f \in B_{p, q}^{s}(\Omega)$ means that there exists some $\tilde{f} \in B_{p, q}^{s}\left(\mathbb{R}^{n}\right)$ such that for any smooth function $\varphi$ with compact support in $\Omega$ we have

$$
\int_{\Omega} f \varphi \mathrm{d} x=\int_{\mathbb{R}^{n}} \tilde{f} \varphi \mathrm{d} x .
$$

Setting

$$
\|f\|_{B_{p, q}^{s}(\Omega)}:=\inf \|\tilde{f}\|_{B_{p, q}^{s}\left(\mathbb{R}^{n}\right)},
$$

where the infimum is taken over all the functions $\tilde{f}$ such that $(2.6)$ holds, it endows the set $B_{p, q}^{s}(\Omega)$ with a structure of Banach space. We refer here to [5, 7,29$]$.

We also recall that the Besov spaces are a real interpolation family, namely

$$
\left(B_{p, q_{1}}^{s_{1}}(\Omega), B_{p, q_{2}}^{s_{2}}(\Omega)\right)_{\theta, q}=B_{p, q}^{\theta s_{2}+(1-\theta) s_{1}}(\Omega)
$$

whenever $1 \leq p, q, q_{1}, q_{2} \leq \infty, s_{1} \neq s_{2}$ and $\theta \in(0,1)$.

A great deal of our results will be based on the following interpolation property $[3,29]$ :

$$
\left(L_{p}(\Omega), W_{p}^{1}(\Omega)\right)_{s, q}=B_{p, q}^{s}(\Omega) \text { for } 1 \leq q \leq \infty \text { and } s \in(0,1) .
$$

Note that (2.8) defines the Besov spaces in indirect way, which, however, is often very practical.

The following density and duality results will be used several times (see [5,29])

Proposition 2.3. Let $\Omega$ be a Lipschitz domain.

- If $1 \leq p, q<\infty$ and $-1+\frac{1}{p}<s<\frac{1}{p}$ then the set $C_{c}^{\infty}(\Omega)$ of smooth functions with compact support in $\Omega$ is dense in $B_{p, q}^{s}(\Omega)$.

- If $1<p<\infty, 1<q \leq \infty$ and $-1+\frac{1}{p}<s<\frac{1}{p}$, then $B_{p, q}^{s}(\Omega)$ may be identified with the dual space of $B_{p^{\prime}, q^{\prime}}^{-s}(\Omega)$, where $1 / p^{\prime}=1-1 / p$ and $1 / q^{\prime}=1-1 / q$.

We will also use that functions in $B_{p, q}^{s}(\Omega)$ with $s>1 / p$ have a trace at the boundary, see e.g. [29].

Proposition 2.4. If $\Omega$ is a Lipschitz bounded or exterior domain and $1 / p<s$ with $1<p<\infty$ then, for all $q \in[1, \infty]$, the trace operator on $\partial \Omega$ extends continuously from $B_{p, q}^{s}(\Omega)$ to $B_{p, q}^{s-\frac{1}{p}}(\partial \Omega)$.

In order to make the above statement more accurate, we explain what a Besov space on the boundary is. In fact, Besov spaces may be defined on any $r$-dimensional manifold $S$. The idea is to use diffeomorphic maps after localization in order to reduce the definition to that of Besov spaces on $\mathbb{R}^{r}$ (see e.g. [4, 25, 29]). If $s \in(0,1)$ and $p=q \in(1, \infty)$ then the Besov space $B_{p, p}^{s}(S)$ (which will be alternatively denoted by $W_{p}^{s}(S)$ in some places of the paper) may be endowed with the norm

$$
\|u\|_{B_{p, p}^{s}(S)}=\|u\|_{L_{p}(S)}+\|u\|_{\dot{B}_{p, p(s e m i)}^{s}(S)},
$$

where $\|\cdot\|_{\dot{B}_{p, p(s e m i)}^{s}(S)}$ stands for the following homogeneous seminorm:

$$
\|u\|_{\dot{B}_{p, p(s e m i)}^{s}(S)}=\left(\int_{S} \int_{S} \frac{|u(x)-u(y)|^{p}}{|x-y|^{r+s p}} \mathrm{~d} x \mathrm{~d} y\right)^{1 / p} .
$$

The above double integral may be also used to define the homogeneous Besov spaces $\dot{B}_{p, p}^{s}(\Omega)$ and $\dot{B}_{p, p}^{s}(\partial \Omega)$. We shall be careful with this definition, since it has different properties for different types of sets $S$. The main examples are $S=\mathbb{R}^{n}$ and $S=\Omega$, where $\Omega$ is a subset of $\mathbb{R}^{n}$ with boundaries (bounded, or the halfspace).

More precisely, for $0<s<\frac{1}{p}$ and $1<p<\infty$, we define the space $\dot{B}_{p, p}^{s}(\Omega)$ as

$$
\dot{B}_{p, p}^{s}(\Omega)=\overline{C_{c}^{\infty}(\Omega)}\|\cdot\|_{\dot{B}_{p, p}^{s}\left(\mathbb{R}^{n}\right)} .
$$


This point requires some precision. The quantity (2.10) is indeed not a norm, except for the case $S=\mathbb{R}^{n}$.

This follows from the fact (2.10) does not see constants. Thus the best solution is to consider the extension of a function onto the whole space; we call it $E f$ for a function $f$ defined on $\Omega$. Then we put

$$
\|f\|_{\dot{B}_{p, p}^{s}(\Omega)} \cong\|E f\|_{\dot{B}_{p, p}^{s}\left(\mathbb{R}^{n}\right)} \cong\|f\|_{\dot{B}_{p, p(s e m i)}^{s}(\Omega)}+\|f\|_{L_{q}(\Omega)}
$$

with $\frac{1}{p}-\frac{1}{q}=\frac{s}{n}$ (for $s \in\left(0, \frac{1}{p}\right)$ the number $q$ is finite). The r.h.s. is the inhomogeneous norm, hence it allows to extend the function on the whole space, and this fact explains relation (2.12).

For such small $s$ the above space is a Banach space, additionally we obtain the spaces $\dot{B}_{p, p}^{s}(\Omega)$ for $-1+\frac{1}{p}<s<0$ by duality: we set

$$
\dot{B}_{p, p}^{s}(\Omega):=\left(\dot{B}_{p^{\prime}, p^{\prime}}^{-s}(\Omega)\right)^{*} .
$$

The remaining spaces $B_{p, q}^{s}(\Omega)$ for $1<p<\infty, 1 \leq q \leq \infty$ and $-1+1 / p<s<1 / p$ may be defined by interpolation according to the following relation:

$$
\left(\dot{B}_{p, p}^{s_{1}}(\Omega), \dot{B}_{p, p}^{s_{2}}(\Omega)\right)_{\theta, q}=\dot{B}_{p, q}^{s}(\Omega) .
$$

We just have to fix some $-1+1 / p<s_{1}<s_{2}<1 / p$ and take $\theta \in(0,1)$ such that $s=\theta s_{2}+(1-\theta) s_{1}$. To have the full picture of possible definitions of Besov space we refer to Triebel's book [29].

We now introduce some auxiliary results. First let us show the following

Lemma 2.5. Let $\Omega \in C^{1,0}$ be an exterior domain, $\boldsymbol{f} \in L_{p}\left(\Omega ; \mathbb{R}^{3}\right)$ and $\operatorname{div} \boldsymbol{f}=0$ in $\mathcal{D}^{\prime}(\Omega)$ and $\vec{n} \cdot \boldsymbol{f}=0$ at $\partial \Omega$ (in the sense of $W_{p}^{-1 / p}(\partial \Omega)$ ). Then there exists a sequence $\boldsymbol{f}_{\epsilon} \in C_{c}^{\infty}\left(\bar{\Omega} ; \mathbb{R}^{3}\right)$ such that

$$
\boldsymbol{f}_{\epsilon} \rightarrow \boldsymbol{f} \text { in } L_{p}\left(\Omega ; \mathbb{R}^{3}\right) \text { as } \epsilon \rightarrow 0, \quad \operatorname{div} \boldsymbol{f}_{\epsilon}=0 \text { and } \operatorname{supp} \boldsymbol{f}_{\epsilon} \subset B_{\frac{1}{\epsilon}}(0) .
$$

Proof. Note that due to the assumption $\vec{n} \cdot \boldsymbol{f}=0$ at the boundary it is possible to extend $\boldsymbol{f}$ on the whole space by zero, preserving its divergence free property. Let us denote the extension by $\boldsymbol{F}$. Next, we take $R$ sufficiently large and examine the function $\boldsymbol{F} \chi_{B_{R}}$. Indeed, in general, $\operatorname{div} \boldsymbol{F} \chi_{B_{R}} \neq 0$, thus we correct this truncation.

The trace $\left.\vec{n} \cdot \boldsymbol{F}\right|_{\partial B_{R}(0)}$ may not be zero, hence we ought to solve the following problem

$$
\begin{array}{ll}
\Delta \phi=0 & \text { in } B_{R}(0), \\
\frac{\partial \phi}{\partial \vec{n}}=\vec{n} \cdot \boldsymbol{F} & \text { at } \partial B_{R}(0) .
\end{array}
$$

Since $\operatorname{div} \boldsymbol{F}=0$ in $\mathbb{R}^{3}$, and $\partial B_{R}(0)$ may be treated as a boundary of sets $B_{R}(0)$ or $\mathbb{R}^{3} \backslash B_{R}(0)$, we find

$$
\|\vec{n} \cdot \boldsymbol{F}\|_{\dot{W}_{p}^{-1 / p}\left(\partial B_{R}(0)\right)} \leq C\|\boldsymbol{F}\|_{L_{p}\left(\mathbb{R}^{3} \backslash B_{R}\right)} .
$$

As $\boldsymbol{F} \in L_{p}\left(\mathbb{R}^{3} ; \mathbb{R}^{3}\right)$, so the r.h.s. of (2.16) goes to zero as $R \rightarrow \infty$. Solution to system (2.15) gives that

$$
\|\nabla \phi\|_{L_{p}\left(B_{R}(0)\right)} \leq C\|\vec{n} \cdot \boldsymbol{F}\|_{\dot{W}_{p}^{-1 / p}\left(\partial B_{R}(0)\right)} .
$$

So we take

$$
\boldsymbol{F}_{R}= \begin{cases}\boldsymbol{F}-\nabla \phi & \text { for }|x| \leq R \\ 0 & \text { for }|x|>R .\end{cases}
$$

Then $\operatorname{div} \boldsymbol{F}_{R}=0$ in $\mathbb{R}^{3}$, since by definition $\vec{n} \cdot \boldsymbol{F}_{R}=0$ at $\partial B_{R}(0)$.

Additionally, by (2.17) we find

$$
\left\|\boldsymbol{F}_{R}-\boldsymbol{F}\right\|_{L_{p}\left(\mathbb{R}^{3}\right)} \rightarrow 0 \text { as } R \rightarrow \infty .
$$

In general $\boldsymbol{F}_{R}$ may not be smooth, so we mollify it introducing

$$
\boldsymbol{F}_{R, \epsilon}=\omega_{\epsilon} * \boldsymbol{F}_{R}, \text { then } \operatorname{div} \boldsymbol{F}_{R, \epsilon}=0 \text { in } \mathbb{R}^{3}
$$

with $\omega_{\epsilon}$ the standard mollifier $\left(\omega_{\epsilon} \rightarrow \delta\right.$ in $\mathcal{D}^{\prime}\left(\mathbb{R}^{3}\right)$ as $\left.\epsilon \rightarrow 0^{+}\right)$. To finish, we put

$$
\boldsymbol{f}_{\epsilon}=\boldsymbol{F}_{R(\epsilon), \epsilon} \mid \Omega,
$$

where $R(\epsilon) \rightarrow+\infty$ for $\epsilon \rightarrow 0^{+}$. Then for a suitable choice of $R(\epsilon)$, function $\boldsymbol{f}_{\epsilon}$ fulfills (2.14). 
The a priori estimate of solutions to the second order elliptic problems will fundamentally depend on results for the Laplace operator in the halfspace.

$$
\begin{aligned}
\Delta \boldsymbol{e} & =\boldsymbol{f} & & \text { in } \mathbb{R}_{+}^{3}, \\
e_{1} & =0, e_{2}=0, e_{3,3}=g & & \text { on } \mathbb{R}^{2} \times\left\{x_{3}=0\right\} .
\end{aligned}
$$

Lemma 2.6. Let $\boldsymbol{f} \in L_{p}\left(\mathbb{R}_{+}^{3} ; \mathbb{R}^{3}\right)$. Then

$$
\left\|\nabla^{2} \boldsymbol{e}\right\|_{L_{p}\left(\mathbb{R}_{+}^{3}\right)} \leq C\left(\|\boldsymbol{f}\|_{L_{p}\left(\mathbb{R}_{+}^{3}\right)}+\|g\|_{\dot{W}_{p}^{1-1 / p}\left(\mathbb{R}^{2}\right)}\right) .
$$

Let $\boldsymbol{f} \in \dot{B}_{p, q}^{s}\left(\mathbb{R}_{+}^{3} ; \mathbb{R}^{3}\right)$ for $-1+\frac{1}{p}<s<\frac{1}{p}$. Then

$$
\left\|\nabla^{2} \boldsymbol{e}\right\|_{\dot{B}_{p, q}^{s}\left(\mathbb{R}_{+}^{3}\right)} \leq C\left(\|\boldsymbol{f}\|_{\dot{B}_{p, q}^{s}\left(\mathbb{R}_{+}^{3}\right)}+\|g\|_{\dot{B}_{p, q}^{s+1-1 / p}\left(\mathbb{R}^{2}\right)}\right) .
$$

Proof. In order to prove inequalities (2.23) and (2.24), let us observe that they follow from the results for scalar equations for the model problems in the half space. The boundary condition in that case can be split into three independent parts.

Due to the basic properties of the Laplace operator we are able to solve model problem in the half space via symmetry. In the case of Dirichlet boundary conditions we admit skew-symmetric extension of the problem getting a system in the whole space, then the standard Marcinkiewicz multiplier theorem $[19,29]$ yields that solution to

$$
\begin{aligned}
\Delta u=h & \text { in } \mathbb{R}_{+}^{3} \\
\left.u\right|_{x_{3}=0}=0 & \text { on } \mathbb{R}^{2}
\end{aligned}
$$

fulfills

$$
\left\|\nabla^{2} u\right\|_{L_{p}\left(\mathbb{R}_{+}^{3}\right)} \leq C\|h\|_{L_{p}\left(\mathbb{R}_{+}^{3}\right)}
$$

moreover, the function $u$ is locally integrable. Additionally, if $h \in \dot{B}_{p, q}^{s}\left(\mathbb{R}_{+}^{3}\right)$ with $-2+\frac{1}{p}<s<\frac{1}{p}$, we extend the function in the skew-symmetric way keeping the desired class of regularity. Namely, the skew-symmetric subspace of $\dot{B}_{p, q}^{s}\left(\mathbb{R}^{3}\right)$ for such $s$ is a well defined subspace of the dual space to $\dot{B}_{p^{\prime}, q^{\prime}}^{-s}\left(\mathbb{R}^{3}\right)$. Thanks to the symmetry we gain one derivative more. See details in [7] and [21]. Then we get

$$
\left\|\nabla^{2} u\right\|_{\dot{B}_{p, q}^{s}\left(\mathbb{R}_{+}^{3}\right)} \leq C\|h\|_{\dot{B}_{p, q}^{s}\left(\mathbb{R}_{+}^{3}\right)} .
$$

A weaker result we get for the Neumann problem:

$$
\begin{aligned}
\Delta u & =h \text { in } \mathbb{R}_{+}^{3}, \\
\left.u_{, x_{3}}\right|_{x_{3}}=0 & =g \text { on } \mathbb{R}^{2} .
\end{aligned}
$$

We use symmetric extension to $\mathbb{R}^{3}$ for $g=0$ and extension of $g$ to $\mathbb{R}_{+}^{3}$ if $g \neq 0$. In the case of the simple $L_{p}$ spaces with the compatibility condition $\int_{\mathbb{R}_{+}^{3}} h \mathrm{~d} x=\int_{\mathbb{R}^{2}} g \mathrm{~d} x^{\prime}$ (if they are defined) we get

$$
\left\|\nabla^{2} u\right\|_{L_{p}\left(\mathbb{R}_{+}^{3}\right)} \leq C\left(\|h\|_{L_{p}\left(\mathbb{R}_{+}^{3}\right)}+\|g\|_{\dot{W}_{p}^{1-\frac{1}{p}}\left(\mathbb{R}^{2}\right)}\right)
$$

and for $h \in \dot{B}_{p, q}^{s}\left(\mathbb{R}_{+}^{3}\right)$ with $-1+\frac{1}{p}<s<\frac{1}{p}$ (the condition on $s$ is more restrictive than for the Dirichlet boundary conditions)

$$
\left\|\nabla^{2} u\right\|_{\dot{B}_{p, q}^{s}\left(\mathbb{R}_{+}^{3}\right)} \leq C\left(\|h\|_{\dot{B}_{p, q}^{s}\left(\mathbb{R}_{+}^{3}\right)}+\|g\|_{\dot{B}_{p, q}^{s+1-1 / p}\left(\mathbb{R}^{2}\right)}\right) .
$$

Collecting (2.26), (2.27) and (2.29), (2.30) we get (2.23) and (2.24), respectively.

The last two lemmas concern multiplication in Besov space in the case as one of the functions has a compact support.

Lemma 2.7. If $f \in W_{\infty}^{1}\left(\mathbb{R}^{3}\right), g \in \dot{B}_{3,1}^{0}\left(\mathbb{R}^{3}\right)$ and $\operatorname{supp} g \subset B_{\lambda}(0)$, then

$$
\|f g\|_{\dot{B}_{3,1}^{0}\left(\mathbb{R}^{3}\right)} \leq C\left(\|f\|_{L_{\infty}\left(\mathbb{R}^{3}\right)}+\lambda\|f\|_{\dot{W}_{\infty}^{1}\left(\mathbb{R}^{3}\right)}\right)\|g\|_{\dot{B}_{3,1}^{0}\left(\mathbb{R}^{3}\right)} .
$$


Proof. The proof of the above result will be a consequence of the interpolation theorem and two estimates proved for the Besov spaces $\dot{B}_{p, p}^{s}(\Omega)$. We refer also to [25].

Let $1<p<\infty$ and $0<s<\frac{1}{p}$, then

$$
\|f g\|_{\dot{B}_{p, p}^{s}\left(\mathbb{R}^{3}\right)} \leq C\left(\|f\|_{L_{\infty}\left(\mathbb{R}^{3}\right)}+\lambda\|f\|_{\dot{W}_{\infty}^{1}\left(\mathbb{R}^{3}\right)}\right)\|g\|_{\dot{B}_{p, p}^{s}\left(\mathbb{R}^{3}\right)} .
$$

In order to prove (2.32) we apply the integral definition of the Besov spaces (2.12). Because of the support of $g$, we conclude that $f g \in \dot{B}_{p, p}^{s}\left(B_{\lambda}(0)\right)$. Hence first we consider the $\dot{B}_{p, p(s e m i)}^{s}$ norm. We have

$$
\begin{aligned}
\|f g\|_{\dot{B}_{p, p(s e m i)}^{s}\left(B_{\lambda}(0)\right)}^{p} & =\int_{B_{\lambda}(0)} \mathrm{d} x \int_{B_{\lambda}(0)} \mathrm{d} x^{\prime} \frac{\left|(f g)(x)-(f g)\left(x^{\prime}\right)\right|^{p}}{\left|x-x^{\prime}\right|^{3+s p}} \\
& \leq \int_{B_{\lambda}(0)} \mathrm{d} x \int_{B_{\lambda}(0)} \mathrm{d} x^{\prime}\left(\frac{\left|f(x)-f\left(x^{\prime}\right)\right|^{p}|g(x)|^{p}}{\left|x-x^{\prime}\right|^{3+s p}}+\frac{\left|f\left(x^{\prime}\right)\right|^{p}\left|g(x)-g\left(x^{\prime}\right)\right|^{p}}{\left.\left.\left|x-x^{\prime}\right|^{3+s p}\right)\right)}\right. \\
& =I_{1}+I_{2} .
\end{aligned}
$$

We consider the first integral

$$
\begin{aligned}
I_{1} & \leq C\|f\|_{\dot{W}_{\infty}^{1}\left(\mathbb{R}^{3}\right)}^{p} \int_{B_{\lambda}(0)} \mathrm{d} x|g(x)|^{p} \int_{B_{\lambda}(0)} \frac{\mathrm{d} x^{\prime}}{\left|x-x^{\prime}\right|^{3+(s-1) p}} \\
& \leq C\|f\|_{\dot{W}_{\infty}^{1}\left(\mathbb{R}^{3}\right)}^{p} \int_{B_{\lambda}(0)}|g(x)|^{p} \int_{0}^{2 \lambda} r^{(1-s) p-1} \mathrm{~d} r \\
& \leq C\|f\|_{\dot{W}_{\infty}^{1}\left(\mathbb{R}^{3}\right)}^{p} \lambda^{p(1-s)}\left|B_{\lambda}(0)\right|^{1-p / p^{*}}\|g\|_{L_{p^{*}}\left(\mathbb{R}^{3}\right)}^{p} \\
& \leq C \lambda^{p}\|f\|_{\dot{W}_{\infty}^{1}\left(\mathbb{R}^{3}\right)}^{p}\|g\|_{\dot{B}_{p, p}^{s}\left(\mathbb{R}^{3}\right)}^{p}
\end{aligned}
$$

with $\frac{3}{s}\left(\frac{1}{p}-\frac{1}{p^{*}}\right)=1$; note that $p^{*}$ is finite, because $s<\frac{1}{p}$.

The second integral from (2.33) is estimated as follows

$$
\begin{aligned}
I_{2} & \leq C\|f\|_{L_{\infty}\left(\mathbb{R}^{3}\right)}^{p} \int_{B_{\lambda}(0)} \mathrm{d} x \int_{B_{\lambda}(0)} \mathrm{d} x^{\prime} \frac{\left|g(x)-g\left(x^{\prime}\right)\right|^{p}}{\left|x-x^{\prime}\right|^{3+s p}} \\
& \leq C\|f\|_{L_{\infty}\left(\mathbb{R}^{3}\right)}^{p}\|g\|_{\dot{B}_{p, p}^{s}\left(\mathbb{R}^{3}\right)}^{p} .
\end{aligned}
$$

Now we check

$$
\|f g\|_{L_{q}(\Omega)} \leq\|f\|_{L_{\infty}\left(\mathbb{R}^{3}\right)}\|g\|_{L_{q}\left(\mathbb{R}^{3}\right)} \leq C\|f\|_{L_{\infty}\left(\mathbb{R}^{3}\right)}\|g\|_{\dot{B}_{p, p}^{s}\left(\mathbb{R}^{3}\right)}
$$

with $\frac{1}{p}-\frac{1}{q}=\frac{s}{3}$. This finishes the proof of $(2.32)$.

Next we prove that if $f \in W_{\infty}^{1}\left(\mathbb{R}^{3}\right)$ and $g \in \dot{B}_{p, p}^{-s}\left(B_{\lambda}(0)\right)$ with $|s|<1-\frac{1}{p}$,

$$
\|f g\|_{\dot{B}_{p, p}^{-s}\left(\mathbb{R}^{3}\right)} \leq C\left(\|f\|_{L_{\infty}\left(\mathbb{R}^{3}\right)}+\lambda\|f\|_{\dot{W}_{\infty}^{1}\left(\mathbb{R}^{3}\right)}\right)\|g\|_{\dot{B}_{p, p}^{-s}\left(\mathbb{R}^{3}\right)} .
$$

To show (2.37) we just reduce the proof to an application of (2.32) and duality argument. By the definition of spaces with negative power, we find

$$
\|f g\|_{\dot{B}_{p, p}^{-s}\left(\mathbb{R}^{3}\right)}=\sup _{h} \int f g h \mathrm{~d} x \leq C\left(\|f\|_{L_{\infty}\left(\mathbb{R}^{3}\right)}+\lambda\|f\|_{\dot{W}_{\infty}^{1}\left(\mathbb{R}^{3}\right)}\right)\|g\|_{\dot{B}_{p, p}^{-s}\left(\mathbb{R}^{3}\right)}\|h\|_{\dot{B}_{q, q}^{s}\left(\mathbb{R}^{3}\right)},
$$

where the supremum is taken over all $h \in \dot{B}_{q, q}^{s}\left(\mathbb{R}^{3}\right)$ with the norm less or equal 1 . As $s<1-\frac{1}{p}=\frac{1}{q}$, we may consider only functions supported in the ball. Taking into account results (2.32) and (2.37), using (2.13) we find (2.31). Lemma 2.7 is proved. 
Lemma 2.8. Let $g \in \dot{B}_{3,1}^{1}\left(\mathbb{R}^{3}\right)$ such that $\operatorname{supp} g \subset B_{\lambda}(0)$, then $g \in \dot{B}_{3,1}^{0}\left(\mathbb{R}^{3}\right)$ and

$$
\|g\|_{\dot{B}_{3,1}^{0}\left(\mathbb{R}^{3}\right)} \leq C \lambda\|g\|_{\dot{B}_{3,1}^{1}\left(\mathbb{R}^{3}\right)} .
$$

Proof. As usual in this limit case we will apply indirect technique of interpolations. Let us assume that in the below paragraph $g \in C_{0}^{\infty}\left(B_{\lambda}(0)\right)$; hence it may be extended by zero to the whole $\mathbb{R}^{3}$.

We show that if $g \in \dot{B}_{p, p}^{1+s}\left(\mathbb{R}^{3}\right)$, then $g \in \dot{B}_{p, p}^{s}\left(\mathbb{R}^{3}\right)$ and

$$
\|g\|_{\dot{B}_{p, p}^{s}\left(\mathbb{R}^{3}\right)} \leq C \lambda\|g\|_{\dot{B}_{p, p}^{1+s}\left(\mathbb{R}^{3}\right)}
$$

for $1<p<\infty$.

Note that we easily find the following bounds:

$$
\|g\|_{\dot{W}_{p}^{1}\left(\mathbb{R}^{3}\right)} \leq C \lambda\|g\|_{\dot{W}_{p}^{2}\left(\mathbb{R}^{3}\right)}, \quad\|g\|_{L_{p}\left(\mathbb{R}^{3}\right)} \leq C \lambda\|g\|_{\dot{W}_{p}^{1}\left(\mathbb{R}^{3}\right)} .
$$

Recall that

$$
\dot{B}_{p, p}^{s}\left(\mathbb{R}^{3}\right)=\left(L_{p}\left(\mathbb{R}^{3}\right), \dot{W}_{p}^{1}\left(\mathbb{R}^{3}\right)\right)_{s, p}, \quad \dot{B}_{p, p}^{1+s}\left(\mathbb{R}^{3}\right)=\left(\dot{W}_{p}^{1}\left(\mathbb{R}^{3}\right), \dot{W}_{p}^{2}\left(\mathbb{R}^{3}\right)\right)_{s, p}
$$

for $s \in(0,1)$. Here spaces $\dot{W}_{p}^{t}$ and $\dot{B}_{p, p}^{t}$ are closures of smooth functions with support bounded in $B_{\lambda}(0)$. From (2.41) and (2.42) we find (2.40). Subsequently we consider the case with negative powers. Here we will assume that $s$ is near zero. Let $g \in \dot{B}_{p, p}^{1-s}\left(\mathbb{R}^{3}\right)$; we want to show that $g \in \dot{B}_{p, p}^{-s}\left(\mathbb{R}^{3}\right)$.

We have by the dual definition of the norm

$$
\|g\|_{\dot{B}_{p, p}^{-s}\left(\mathbb{R}^{3}\right)}=\sup _{h} \int_{\mathbb{R}^{3}} g h \mathrm{~d} x,
$$

where the supremum is taken over all $h \in \dot{B}_{q, q}^{s}\left(B_{\lambda}(0)\right) \cap\left\{\int_{B_{\lambda}(0)} h d x=0\right\}$ with the norm less than one. Here we remind that the support of $g$ is in $B_{\lambda}(0)$ and the taken assumption gives $\int_{B_{\lambda}(0)} g \mathrm{~d} x=0$. Next, let us observe that considering two imbeddings

$$
L_{p}\left(B_{\lambda}(0)\right) \hookrightarrow L_{p}\left(B_{\lambda}(0)\right) \text { and } \dot{W}_{p}^{1}\left(B_{\lambda}(0)\right) \hookrightarrow L_{p}\left(B_{\lambda}(0)\right)
$$

the interpolation gives us

$$
\left(L_{p}\left(B_{\lambda}(0)\right), \dot{W}_{p}^{1}\left(B_{\lambda}(0)\right)\right)_{s, p} \hookrightarrow L_{p}\left(B_{\lambda}(0)\right),\|g\|_{L_{p}\left(B_{\lambda}(0)\right)} \leq C \lambda^{1-s}\|g\|_{\dot{B}_{p, p}^{1-s}\left(B_{\lambda}(0)\right)} .
$$

On the other hand, $h \in L_{q^{*}}\left(B_{\lambda}(0)\right)$ with $\frac{3}{s}\left(\frac{1}{q}-\frac{1}{q^{*}}\right)=1$. As $s<\frac{3}{q}=3\left(1-\frac{1}{p}\right)$, then $q^{*}$ is finite. Hence we get

$$
\begin{aligned}
\|g\|_{\dot{B}_{p, p}^{-s}\left(\mathbb{R}^{3}\right)} & \leq C \lambda^{1-s}\|g\|_{\dot{B}_{p, p}^{1-s}\left(\mathbb{R}^{3}\right)}\|h\|_{L_{q}\left(B_{\lambda}(0)\right)} \\
& \leq C \lambda^{1-s}\|g\|_{\dot{B}_{p, p}^{1-s}\left(\mathbb{R}^{3}\right)}\left|B_{\lambda}(0)\right|^{1 / q-1 / q^{*}}\|h\|_{L_{q^{*}}\left(B_{\lambda}(0)\right)} \\
& \leq C \lambda\|g\|_{\dot{B}_{p, p}^{1-s}\left(\mathbb{R}^{3}\right)}\|h\|_{\dot{B}_{p, p}^{1-s}\left(B_{\lambda}(0)\right)}
\end{aligned}
$$

We proved

$$
\|g\|_{\dot{B}_{p, p}^{-s}\left(\mathbb{R}^{3}\right)} \leq C \lambda\|g\|_{\dot{B}_{p, p}^{1-s}\left(\mathbb{R}^{3}\right)} \text { for } s<3(1-1 / p)
$$

Taking (2.40) and (2.47) together, the interpolation theorem (see (2.13)) yields

$$
\|g\|_{\dot{B}_{3,1}^{0}\left(\mathbb{R}^{3}\right)} \leq C \lambda\|g\|_{\dot{B}_{3,1}^{1}\left(\mathbb{R}^{3}\right)} .
$$

\section{Basic Existence in the $L_{2}$-Framework}

The first part which needs some explanation is the existence result for (a smooth compactly supported) right-hand side in the Hilbert space setting. We want to use this result in order to establish existence of solutions (for smooth and compactly supported r.h.s). This point is very important, since it enables to construct a sequence of approximative solutions for general right-hand sides. We aim at proving the following result: 
Theorem 3.1. Let $\Omega$ be as in Theorem 1.4. Let $\boldsymbol{f} \in L_{2}\left(\Omega ; \mathbb{R}^{3}\right)$, $\operatorname{div} \boldsymbol{f}=0$ in $\mathcal{D}^{\prime}(\Omega)$, in addition let $\boldsymbol{f}$ have a compact support. Then there exists a unique solution to (2.1) such that $\boldsymbol{e} \in W_{2(\text { loc })}^{1}\left(\Omega ; \mathbb{R}^{3}\right)$ and

$$
\|\nabla \boldsymbol{e}\|_{L_{2}(\Omega)}+\left\|\left.\boldsymbol{e}\right|_{K}\right\|_{L_{2}(K)} \leq C\|\boldsymbol{f}\|_{L_{2}(\Omega)},
$$

where $C$ depends on the measure of support of $\boldsymbol{f}$.

Section 3 is devoted to the proof of this theorem. To fix the functional setting, we introduce

$$
\widetilde{H}=\left\{\boldsymbol{u} \in C_{c}^{2}\left(\bar{\Omega} ; \mathbb{R}^{3}\right), \operatorname{div} \boldsymbol{u}=0 \text { in } \Omega \text { and } \boldsymbol{u} \cdot \vec{\tau}_{k}=0, k=1,2 \text {, at } \partial \Omega\right\} .
$$

We first start with the case $\partial \Omega \in C^{2}$, to avoid certain technical steps. We return to the case of less regular boundary at the end of this section. We have

Lemma 3.2. Let $\Omega$ be as above with $\partial \Omega \in C^{2}$. Then there exists $C>0$, depending on the measure of the compact subset $K \subset \bar{\Omega}$, supp $\boldsymbol{e} \subset K$, such that for any $\boldsymbol{e} \in \widetilde{H}$ the following inequality holds

$$
\|\nabla \boldsymbol{e}\|_{L_{2}(\Omega)}+\left\|\left.\boldsymbol{e}\right|_{K}\right\|_{L_{2}(K)} \leq C\|\operatorname{rot} \boldsymbol{e}\|_{L_{2}(\Omega)} .
$$

Proof. The first point which should be clarified is the information coming from the boundary condition. Due to the smoothness of $\boldsymbol{e}$ up to the boundary we have $\operatorname{div} \boldsymbol{e}=0$ also on $\partial \Omega$. Taking a fixed point $x_{0} \in \partial \Omega$ we have

$$
-\frac{\partial\left(\boldsymbol{e} \cdot \vec{n}\left(x_{0}\right)\right)}{\partial \vec{n}}=\left(\boldsymbol{e} \cdot \vec{\tau}_{1}\left(x_{0}\right)\right)_{\vec{\tau}_{1}}+\left(\boldsymbol{e} \cdot \vec{\tau}_{2}\left(x_{0}\right)\right)_{\vec{\tau}_{2}} .
$$

Here $\vec{n}\left(x_{0}\right), \vec{\tau}_{1}\left(x_{0}\right), \vec{\tau}_{2}\left(x_{0}\right)$ are fixed constant vectors defined by the frame at tangent space at point $x_{0}$.

From $\boldsymbol{e} \cdot \vec{\tau}_{k}=0$ we find

$$
\left(\boldsymbol{e} \cdot \vec{\tau}_{k}\right)_{\vec{\tau}_{k}}=0, \text { so } e_{\vec{\tau}_{k}} \cdot \vec{\tau}_{k}+e \cdot \vec{\tau}_{k, \vec{\tau}_{k}}=0 \text { at } \partial \Omega
$$

looking at the point $x_{0}$

$$
\boldsymbol{e}_{\vec{\tau}_{k}} \cdot \vec{\tau}_{k}=-\chi_{k}\left(x_{0}\right) \boldsymbol{e} \cdot \vec{n}
$$

where $\vec{\tau}_{k, \vec{\tau}_{k}}=\chi_{k} \cdot \vec{n}$ (Frenet formula).

So we have

$$
\frac{\partial(\boldsymbol{e} \cdot \vec{n})}{\partial \vec{n}}=\boldsymbol{e} \cdot \vec{n}\left(\chi_{1}\left(x_{0}\right)+\chi_{2}\left(x_{0}\right)\right) .
$$

Recalling the well known identity $\operatorname{rot} \operatorname{rot} \boldsymbol{e}=\nabla \operatorname{div} \boldsymbol{e}-\Delta \boldsymbol{e}$, multiplying it by $\boldsymbol{e}$, integrating over $\Omega$ and performing integration by parts we get

$$
\int_{\Omega}|\nabla \boldsymbol{e}|^{2} \mathrm{~d} x=\int_{\Omega}(\operatorname{rot} \boldsymbol{e})^{2} \mathrm{~d} x+\int_{\partial \Omega} \frac{\partial \boldsymbol{e}}{\partial \vec{n}} \cdot \boldsymbol{e} \mathrm{d} \sigma .
$$

Hence by (3.7) and the condition for the tangent value at the boundary we find

$$
\begin{aligned}
\int_{\partial \Omega} \frac{\partial \boldsymbol{e}}{\partial \vec{n}} \cdot \boldsymbol{e} \mathrm{d} \sigma & =\int_{\partial \Omega}\left[\left(\frac{\partial \boldsymbol{e}}{\partial \vec{n}} \cdot \vec{n}\right)(\boldsymbol{e} \cdot \vec{n})+\sum_{k=1}^{n}\left(\frac{\partial \boldsymbol{e}}{\partial \vec{n}} \cdot \vec{\tau}_{k}\right)\left(\boldsymbol{e} \cdot \overrightarrow{\tau_{k}}\right)\right] \mathrm{d} \sigma \\
& =\int_{\partial \Omega}\left(\frac{\partial \boldsymbol{e}}{\partial \vec{n}} \cdot \vec{n}\right)(\boldsymbol{e} \cdot \vec{n}) \mathrm{d} \sigma=\int_{\partial \Omega}\left(\chi_{1}+\chi_{2}\right)(\boldsymbol{e} \cdot \vec{n})^{2} \mathrm{~d} \sigma .
\end{aligned}
$$

Here we assume that $\chi_{k}$ is bounded $\left(\partial \Omega \in C^{2}\right)$, but we return to this issue later.

The above analysis yields the following relation

$$
\|\nabla \boldsymbol{e}\|_{L_{2}(\Omega)} \leq C\left(\|\operatorname{rot} \boldsymbol{e}\|_{L_{2}(\Omega)}+\left\|\left.\boldsymbol{e}\right|_{\partial \Omega}\right\|_{L_{2}(\partial \Omega)}\right) .
$$

The last part can be substituted by $\left\|\left.\boldsymbol{e}\right|_{K}\right\|_{L_{2}(K)}$ by an elementary application of the trace theorem $\|\boldsymbol{e}\|_{L_{2}(\partial \Omega)} \leq \epsilon\|\nabla \boldsymbol{e}\|_{L_{2}(K)}+C(\epsilon)\left\|\left.\boldsymbol{e}\right|_{K}\right\|_{L_{2}(K)}$, i.e.

$$
\|\nabla \boldsymbol{e}\|_{L_{2}(\Omega)} \leq C\left(\|\operatorname{rot} \boldsymbol{e}\|_{L_{2}(\Omega)}+\left\|\left.\boldsymbol{e}\right|_{K}\right\|_{L_{2}(K)}\right) .
$$

Next, let us show

$$
\left\|\left.\boldsymbol{e}\right|_{K}\right\|_{L_{2}(K)} \leq C\|\operatorname{rot} \boldsymbol{e}\|_{L_{2}(\Omega)}
$$


Assume that (3.12) is not true, so there exists a sequence $\left\{\boldsymbol{e}_{k}\right\}$ such that

$$
\left\|\left.\boldsymbol{e}_{k}\right|_{K}\right\|_{L_{2}(K)}>k\left\|\operatorname{rot} \boldsymbol{e}_{k}\right\|_{L_{2}(\Omega)} .
$$

By (3.11) and (3.13) we are able to ensure that

$$
\left\|\left.\boldsymbol{e}_{k}\right|_{K}\right\|_{L_{2}(K)}=1
$$

so

$$
\left\|\nabla \boldsymbol{e}_{k}\right\|_{L_{2}(\Omega)} \leq C \frac{k+1}{k}\left\|\left.\boldsymbol{e}_{k}\right|_{K}\right\|_{L_{2}(K)} \leq \text { const } .
$$

By (3.14) and (3.15) there exists a function $\boldsymbol{e}^{*}$ such that up to a subsequence

$$
\nabla \boldsymbol{e}_{k} \rightarrow \nabla \boldsymbol{e}^{*} \text { weakly in } L_{2}\left(\Omega ; \mathbb{R}^{3 \times 3}\right) \text {. }
$$

But (3.13) yields that rot $\boldsymbol{e}^{*} \equiv 0$, moreover $\int_{\partial \Omega} \boldsymbol{e}_{\boldsymbol{k}} \cdot \vec{n} \mathrm{~d} \sigma=0$, hence the limit $\boldsymbol{e}^{*}$ fulfills (in the weak sense, using the well-known operator identity $\Delta=\nabla \operatorname{div}$ - rot rot and the boundary conditions)

$$
\begin{aligned}
& \Delta e^{*}=\mathbf{0} \text { in } \Omega, \\
& \boldsymbol{e}^{*} \cdot \vec{\tau}=0 \text { at } \partial \Omega \text {, } \\
& \operatorname{div} \boldsymbol{e}^{*}=0 \text { in } \partial \Omega \text {, } \\
& \int_{\partial \Omega} e^{*} \cdot \vec{n} \mathrm{~d} \sigma=0 .
\end{aligned}
$$

The only solution to (3.17) is $\boldsymbol{e}^{*} \equiv \mathbf{0}$, see Remark 1.2. On the other hand by (3.14) and (3.15) (up to a subsequence) $\left.\left.\boldsymbol{e}_{k}\right|_{K} \rightarrow \boldsymbol{e}^{*}\right|_{K}$ strongly in $L_{2}(K)$, which implies that $\left\|\left.\boldsymbol{e}^{*}\right|_{K}\right\|_{L_{2}(K)}=1$, leading to a contradiction. Hence (3.12) is true.

Due to this lemma we may define

We easily show that

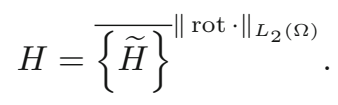

$$
\begin{aligned}
H= & \left\{\boldsymbol{u} \in L_{2(l o c)}\left(\bar{\Omega} ; \mathbb{R}^{3}\right), \nabla \boldsymbol{u} \in L^{2}\left(\Omega ; \mathbb{R}^{3 \times 3}\right) ; \operatorname{div} \boldsymbol{u}=0,\right. \\
& \left.\boldsymbol{u} \cdot \vec{\tau}_{k}=0, k=1,2 \text { at } \partial \Omega, \int_{\partial \Omega} \boldsymbol{u} \cdot \vec{n} \mathrm{~d} \sigma=0\right\}
\end{aligned}
$$

Let us note that the condition $\int_{\partial \Omega} \boldsymbol{u} \cdot \vec{n} \mathrm{~d} \sigma=0$ is for $\boldsymbol{u} \in \widetilde{H}$ fulfilled automatically since the space $\tilde{H}$ consists only of functions with compact support, it is they are zero away a sufficiently large ball. Then the divergence free constraint implies this restriction.

Therefore

Lemma 3.3. Let $\Omega$ be as above with $\partial \Omega \in C^{2}, \boldsymbol{f} \in L_{2}\left(\Omega ; \mathbb{R}^{3}\right) \operatorname{div} \boldsymbol{f}=0$ and $\operatorname{supp} \boldsymbol{f} \in B_{R}(0) \cap \Omega$, then there exists a unique weak solution to (2.1) such that $e \in H$ and the following identity holds

$$
\int_{\Omega} \operatorname{rot} \boldsymbol{e} \cdot \operatorname{rot} \boldsymbol{w} \mathrm{d} x=\int_{\Omega} \boldsymbol{f} \cdot \boldsymbol{w} \mathrm{d} x \text { for all } \boldsymbol{w} \in H ;
$$

additionally

$$
\|\operatorname{rot} \boldsymbol{e}\|_{L_{2}(\Omega)} \leq C\|\boldsymbol{f}\|_{L_{2}(\Omega)}
$$

Proof. We use the Lax-Milgram theorem for a bilinear map

$$
b(\boldsymbol{u}, \boldsymbol{w})=\int_{\Omega} \operatorname{rot} \boldsymbol{e} \cdot \operatorname{rot} \boldsymbol{w} \mathrm{d} x \quad \text { in } H .
$$

By the definition of $H$ the functional $b$ is elliptic, so we shall check whether a functional generated by $f$ is bounded. But $f$ is compactly supported, so

$$
\left|\int_{\Omega} \boldsymbol{f} \cdot \boldsymbol{w} \mathrm{d} x\right| \leq\|\boldsymbol{f}\|_{L_{2}(\Omega)}\|\boldsymbol{w}\|_{L_{2}\left(\Omega \cap B_{R}(0)\right)} \leq C\|\boldsymbol{f}\|_{L_{2}(\Omega)}\|\operatorname{rot} \boldsymbol{w}\|_{L_{2}(\Omega)},
$$

where in the last step we used (3.12). The lemma is proved. 
We want to extend the meaning of the solution, make it a distributional one by replacing $H$ by smooth functions with just boundary condition for the tangent part of functions (zero).

Taking an arbitrary function $\boldsymbol{w} \in C^{\infty}\left(\bar{\Omega} ; \mathbb{R}^{3}\right)$ such that $\boldsymbol{w} \cdot \vec{\tau}_{k}=0$ for $k=1,2$, we define

$$
\widetilde{\boldsymbol{w}}=\boldsymbol{w}-\nabla p,
$$

where $p$ is a solution to the following system

$$
\begin{array}{ll}
\Delta p=\operatorname{div} \boldsymbol{w} & \text { in } \Omega, \\
p=0 & \text { at } \partial \Omega .
\end{array}
$$

Classical solvability via the Lax-Milgram theorem says

$$
\|\nabla p\|_{L_{2}(\Omega)} \leq C\|\boldsymbol{w}\|_{L_{2}(\Omega)} \text { and } \nabla p \cdot \vec{\tau}_{k}=0 \quad k=1,2 .
$$

Thus $\widetilde{\boldsymbol{w}}$ is divergence free and $\widetilde{\boldsymbol{w}} \cdot \vec{\tau}_{k}=0$ for $k=1,2$. Hence

$$
\int_{\Omega} \boldsymbol{f} \cdot \boldsymbol{w} \mathrm{d} x=\int_{\Omega} \boldsymbol{f} \cdot \widetilde{\boldsymbol{w}} \mathrm{d} x+\int_{\Omega} \boldsymbol{f} \cdot \nabla p \mathrm{~d} x=\int_{\Omega} \boldsymbol{f} \cdot \widetilde{\boldsymbol{w}} \mathrm{d} x,
$$

since $\int_{\Omega} \boldsymbol{f} \cdot \nabla p \mathrm{~d} x=0$, by virtue of $\operatorname{div} \boldsymbol{f}=0$ and $\left.p\right|_{\partial \Omega}=0$. Eventually, $\operatorname{rot} \boldsymbol{w}=\operatorname{rot} \widetilde{\boldsymbol{w}}$, thus we proved

Lemma 3.4. Let $\boldsymbol{e}$ be a weak solution given by Lemma 3.3, then $\boldsymbol{e}$ is a distributional solution to (2.1), in particular it holds

$$
\int_{\Omega} \operatorname{rot} \boldsymbol{e} \cdot \operatorname{rot} \boldsymbol{w} \mathrm{d} x=\int_{\Omega} \boldsymbol{f} \cdot \boldsymbol{w} \mathrm{d} x
$$

for $\boldsymbol{w} \in C_{c}^{\infty}\left(\bar{\Omega} ; \mathbb{R}^{3}\right)$ such that

$$
\boldsymbol{w} \cdot \vec{\tau}_{k}=0 \text { at } \partial \Omega
$$

\subsection{Low Regularity Boundary Case}

Let us now consider the case of less regular boundary, i.e. $\partial \Omega \in B_{3,1}^{2-\frac{1}{3}}$. To repeat the scheme of proving existence in the $L_{2}$ framework we meet just one obstacle. We have to consider the boundary integral

$$
\int_{\partial \Omega} \frac{\partial \boldsymbol{e}}{\partial \vec{n}} \cdot \boldsymbol{e} \mathrm{d} \sigma=\int_{\partial \Omega}\left(\chi_{1}+\chi_{2}\right)(\boldsymbol{e} \cdot \vec{n})^{2} \mathrm{~d} \sigma,
$$

but here $\partial \Omega \in B_{3,1}^{2-\frac{1}{3}}$, so $\vec{n} \in C^{\omega(.)}$ and $\chi_{i} \in B_{3,1}^{-1 / 3}$ only. In particular the curvature is a distribution.

On the other hand, $e \in H^{1}\left(K ; \mathbb{R}^{3}\right)$, so to repeat all steps of the proof of Lemma 3.2 it remains to show

$$
\left|\int_{\partial \Omega}\left(\chi_{1}+\chi_{2}\right)(\boldsymbol{e} \cdot \vec{n})^{2} \mathrm{~d} \sigma\right| \leq \epsilon\|\nabla \boldsymbol{e}\|_{L_{2}(K)}^{2}+C\|\boldsymbol{e}\|_{L_{2}(\partial \Omega)}^{2} .
$$

First we answer the question where $\left.(\boldsymbol{e} \cdot \vec{n})^{2}\right|_{\partial \Omega}$ belongs for $\boldsymbol{e} \in H^{1}\left(K ; \mathbb{R}^{3}\right)$.

It is clear that $|\boldsymbol{e}|^{2} \in W_{3 / 2}^{1}(K)$, hence

$$
\left.|\boldsymbol{e}|^{2}\right|_{\partial \Omega} \in W_{3 / 2}^{1 / 3}(\partial \Omega)=B_{3 / 2,3 / 2}^{1 / 3}(\partial \Omega) .
$$

Thus to keep the well posedness of the l.h.s. of (3.30), it is enough to have $\chi_{i} \in B_{3,3}^{-1 / 3}$. In order to show (3.30), we localize it and transform it into the plane $\mathbb{R}^{2}$. So we reduced the issue to the following problem: prove that

$$
\int_{\mathbb{R}^{2}} g a \mathrm{~d} x^{\prime} \leq \epsilon\|\nabla a\|_{L_{3 / 2}\left(\mathbb{R}_{+}^{3}\right)}+C\left\|\left.a\right|_{z_{3}=0}\right\|_{L_{1}\left(\mathbb{R}^{2}\right)}
$$

with supp $g, a \in \overline{B(0, \lambda) \cap \mathbb{R}_{+}^{3}}, g \in B_{3,1}^{-1 / 3}\left(\mathbb{R}^{2}\right)$. Here $g \sim \chi_{1}+\chi_{2}$ and $a \sim|e|^{2}$ at $\partial \Omega$. 
The regularity of $g$ implies that for a given $\epsilon>0$ we are able to find a smooth function $g_{\epsilon}$ such that

$$
\left\|g-g_{\epsilon}\right\|_{B_{3,1}^{-1 / 3}} \leq \epsilon
$$

Since $B_{3,1}^{-1 / 3} \subset B_{3,3}^{-1 / 3}$, we get

$$
\int_{\mathbb{R}^{2}} a\left(g-g_{\epsilon}\right) \mathrm{d} z^{\prime} \leq\left\|g-g_{\epsilon}\right\|_{B_{3,3}^{-1 / 3}}\|a\|_{B_{3 / 2,3 / 2}^{1 / 3}} \leq \epsilon\|a\|_{B_{3 / 2,3 / 2}^{1 / 3}} .
$$

Finally, as $g_{\epsilon}$ is smooth,

$$
\int_{\mathbb{R}^{2}} a g_{\epsilon} \mathrm{d} z^{\prime} \leq C\|a\|_{L_{1}\left(\mathbb{R}^{2}\right)} .
$$

Hence we proved (3.31). Summing the results for the partition of unity for $\partial \Omega$ we get (3.30). Thus we proved Lemma 3.2 assuming $\partial \Omega \in B_{3,1}^{2-\frac{1}{3}}$ only. Taking into account results of Lemmas 3.2, 3.3 and 3.4 we conclude Theorem 3.1.

Remark 3.5. Note that in the case of Theorem 1.3, i.e. $\partial \Omega \in B_{r, q}^{2+s-\frac{1}{r}}$, it may happen that $B_{r, q}^{2+s-\frac{1}{r}}$ is not imbedded into $B_{3,1}^{5 / 3}$. However, in this case we have $B_{r, q}^{2+s-\frac{1}{r}} \hookrightarrow C^{1, a}$ for some $a>0$, hence we get (3.30) also in this case, the proof is even somewhat easier than in the case considered above for the critical regularity space.

\section{The $L_{p}$ Case}

We start with the proof of the global estimate for (2.1) in the standard $L_{p}$ framework. The central point in our considerations is the regularity of the boundary. The structure of our estimates requires that

$$
\partial \Omega \in W_{q}^{2-1 / q}
$$

with $q$ fulfilling the conditions: if $p>3$ then $q=p$ and if $p \leq 3$ then $q>3$ (can be arbitrarily close to 3 ). Recall that in this case, due to the embedding, we know that the local description of the boundary is of the class $C^{1, a}$ for some a positive. Recall also that $W_{q}^{2-1 / q}(\mathcal{O}) \hookrightarrow B_{3,1}^{5 / 3}(\mathcal{O})$ for $\mathcal{O}$ a bounded subset of $\mathbb{R}^{2}$.

First we show the following result

Lemma 4.1. Let $\Omega$ be as in Theorem 1.1, $\boldsymbol{f} \in C_{c}^{\infty}\left(\Omega ; \mathbb{R}^{3}\right)$. Moreover, let $\boldsymbol{e} \in W_{2(\text { loc })}^{1}\left(\Omega ; \mathbb{R}^{3}\right)$ be the solution to (2.1) constructed in Theorem 3.1. Then for any $1<p<\infty$ the following estimate is valid

$$
\left\|\nabla^{2} \boldsymbol{e}\right\|_{L_{p}(\Omega)} \leq C\left(\|\boldsymbol{f}\|_{L_{p}(\Omega)}+\left\|\left.\boldsymbol{e}\right|_{K}\right\|_{W_{p}^{1}(K)}\right) .
$$

Proof. We show (4.2) by localization. Let $\eta^{0}$ be a cut off function "removing" the neighborhood of the boundary of $\Omega$ and $\operatorname{supp} \nabla \eta^{0} \subset K$, see Sect. 2. Then we get in the sense of distributions

$$
\Delta \boldsymbol{E}=2 \nabla \eta^{0} \cdot \nabla \boldsymbol{e}+\boldsymbol{e} \Delta \eta^{0}+\eta^{0} \boldsymbol{f} \quad \text { in } \mathbb{R}^{3}
$$

with $\boldsymbol{E}=\eta^{0} \boldsymbol{e}$. Therefore, immediately

$$
\left\|\nabla^{2} \boldsymbol{E}\right\|_{L_{p}\left(\mathbb{R}^{3}\right)} \leq C\left(\left\|\eta^{0} \boldsymbol{f}\right\|_{L_{p}\left(\mathbb{R}^{3}\right)}+\|\boldsymbol{e}\|_{W_{p}^{1}(K)}\right) .
$$

Note that even though this estimate was shown just formally, it can be easily justified also for our solution which was a priori only in $W_{2(l o c)}^{1}$; indeed, we get our estimate for any $1<p \leq 2$. Hence, taking $p=2$ we know that $\boldsymbol{e}$ belongs to $W_{6(l o c)}^{1}\left(\Omega ; \mathbb{R}^{3}\right)$ and we may repeat the argument above for any $2<p \leq 6$; hence taking $p=6$ we know that $\boldsymbol{e} \in W_{\infty(l o c)}^{1}\left(\Omega ; \mathbb{R}^{3}\right)$ which allows us to consider also $6<p<\infty$. 
Subsequently we consider the case near the boundary. Let $\eta^{0}, \eta^{1}, \ldots, \eta^{N}$ be the partition of unity in $\Omega$, see Sect. 2. Take now $k \in\{1, \ldots, N\}$. As before, we start with a formal argument and then explain its application for $\boldsymbol{e} \in W_{2(l o c)}^{1}(\Omega)$ only. We have for $\boldsymbol{E}=\eta^{k} \boldsymbol{e}$

$$
\begin{array}{ll}
\Delta \boldsymbol{E}=2 \nabla \eta^{k} \cdot \nabla \boldsymbol{e}+\boldsymbol{e} \Delta \eta^{k}+\eta^{k} \boldsymbol{f}=\boldsymbol{f}_{1} & \text { in } \Omega, \\
\boldsymbol{E} \cdot \vec{\tau}_{l}=0 & \text { on } \partial \Omega, \quad l=1,2, \\
\operatorname{div} \boldsymbol{E}=\boldsymbol{e} \cdot \nabla \eta^{k} & \text { on } \partial \Omega .
\end{array}
$$

The above system is supported on the set $\operatorname{supp} \eta^{k}$; we transform it into $\mathbb{R}_{+}^{3}$ getting (cf. Sect. 2)

$$
\begin{array}{ll}
\Delta_{z} Z^{*} \boldsymbol{E}=\left(\Delta_{z}-\Delta_{x}\right) Z^{*} \boldsymbol{E}+Z^{*} \boldsymbol{f}_{1} & \text { in } \mathbb{R}_{+}^{3}, \\
Z^{*} \boldsymbol{E} \cdot \hat{\boldsymbol{e}}_{l}=0 & \text { on } \mathbb{R}^{2}, \\
\left(Z^{*} \boldsymbol{E}\right)_{, z_{3}}^{(3)}=Z^{*}\left(\boldsymbol{e} \cdot \nabla \eta^{k}\right)-\left(\operatorname{div}_{x}-\operatorname{div}_{z}\right) Z^{*} \boldsymbol{E} & \text { on } \mathbb{R}^{2},
\end{array}
$$

where $Z^{*} \boldsymbol{E}=E \circ Z$ and $\hat{\boldsymbol{e}}_{l}, l=1,2$ be the unit vectors in the direction of the $z_{l}$ axis. Therefore (see Lemma 2.6)

$$
\begin{aligned}
\left\|\nabla^{2} Z^{*} \boldsymbol{E}\right\|_{L_{p}\left(\mathbb{R}_{+}^{3}\right) \leq} & C\left(\left\|\left(\Delta_{x}-\Delta_{z}\right) Z^{*} \boldsymbol{E}\right\|_{L_{p}\left(\mathbb{R}_{+}^{3}\right)}+\left\|Z^{*} \boldsymbol{f}_{1}\right\|_{L_{p}\left(\mathbb{R}_{+}^{3}\right)}\right. \\
& \left.+\left\|Z^{*}\left(\boldsymbol{e} \cdot \nabla \eta^{k}\right)\right\|_{W_{p}^{1-\frac{1}{p}}\left(\mathbb{R}^{2}\right)}+\left\|\left(\operatorname{div}_{x}-\operatorname{div}_{z}\right) Z^{*} \boldsymbol{E}\right\|_{W_{p}^{1-\frac{1}{p}}\left(\mathbb{R}^{2}\right)}\right) .
\end{aligned}
$$

However, let us observe that

$$
\left\|\left(\Delta_{x}-\Delta_{z}\right) Z^{*} \boldsymbol{E}\right\|_{L_{p}\left(\mathbb{R}_{+}^{3}\right)} \leq C\left(\lambda^{a}+\epsilon\right)\left\|\nabla^{2} Z^{*} \boldsymbol{E}\right\|_{L_{p}\left(\mathbb{R}_{+}^{3}\right)}+C\left\|Z^{*} \boldsymbol{E}\right\|_{W_{p}^{1}(K)}
$$

In order to prove the above estimate we note that

$$
\left\|\left(\Delta_{x}-\Delta_{z}\right) Z^{*} \boldsymbol{E}\right\|_{L_{p}\left(\mathbb{R}_{+}^{3}\right)} \leq\left\|\left(\operatorname{div}_{x}-\operatorname{div}_{z}\right) \nabla_{x} Z^{*} \boldsymbol{E}\right\|_{L_{p}\left(\mathbb{R}_{+}^{3}\right)}+\left\|\operatorname{div}_{z}\left(\nabla_{x}-\nabla_{z}\right) Z^{*} \boldsymbol{E}\right\|_{L_{p}\left(\mathbb{R}_{+}^{3}\right)} .
$$

To avoid unnecessary notation we write in a symbolic way that

$$
\nabla_{x}=(I d+\boldsymbol{A}) \nabla_{z}, \text { where } \boldsymbol{A}(0)=\mathbf{0} \text { and } \boldsymbol{A} \in W_{q}^{1}\left(B_{\lambda}\left(x^{0}\right) ; \mathbb{R}^{3 \times 3}\right)
$$

with $q=p$ for $p>3$ and $q>3$ for $p \leq n$. Note that $\boldsymbol{A}$ is Hölder continuous due to the embedding theorem. Using the above notation we get

$$
\left\|\left(\Delta_{x}-\Delta_{z}\right) Z^{*} \boldsymbol{E}\right\|_{L_{p}\left(\mathbb{R}_{+}^{3}\right)} \leq C \lambda^{a}\left\|\nabla^{2} Z^{*} \boldsymbol{E}\right\|_{L_{p}\left(\mathbb{R}_{+}^{3}\right)}+C\left\|\nabla \boldsymbol{A} \nabla Z^{*} \boldsymbol{E}\right\|_{L_{p}\left(\mathbb{R}_{+}^{3}\right)}
$$

for some $a>0$. To estimate the last norm we see that for $p>3$ we have

$$
\left\|\nabla Z^{*} \boldsymbol{E}\right\|_{L_{\infty}\left(\mathbb{R}_{+}^{3}\right)} \leq \epsilon\left\|\nabla^{2} Z^{*} \boldsymbol{E}\right\|_{L_{p}\left(\mathbb{R}_{+}^{3}\right)}+C(\epsilon)\left\|Z^{*} \boldsymbol{E}\right\|_{W_{p}^{1}\left(\mathbb{R}_{+}^{3}\right)} .
$$

In the case $p \leq 3$ note that $\frac{1}{p}=\frac{3-p}{3 p}+\frac{1}{3}$. So if $q>3$, there is a room to apply the interpolation inequality to get

$$
\left\|\nabla \boldsymbol{A} \nabla Z^{*} \boldsymbol{E}\right\|_{L_{p}\left(\mathbb{R}_{+}^{3}\right)} \leq \epsilon\left\|\nabla^{2} Z^{*} \boldsymbol{E}\right\|_{L_{p}\left(\mathbb{R}_{+}^{3}\right)}+C(\epsilon)\left\|Z^{*} \boldsymbol{E}\right\|_{W_{p}^{1}\left(\mathbb{R}_{+}^{3}\right)} .
$$

In a similar way we examine the below estimate, note that the considered norm can be viewed as a trace norm, thus the computations for (4.10) stay true and we conclude

$$
\left\|\left(\operatorname{div}_{x}-\operatorname{div}_{z}\right) Z^{*} \boldsymbol{E}\right\|_{W_{p}^{1-\frac{1}{p}}\left(\mathbb{R}^{2}\right)} \leq C\left(\lambda^{a}+\epsilon\right)\left\|\nabla^{2} Z^{*} \boldsymbol{E}\right\|_{L_{p}\left(\mathbb{R}_{+}^{3}\right)}+C\left\|Z^{*} \boldsymbol{E}\right\|_{W_{p}^{1}(K)} .
$$

Finally

$$
\left\|\left.Z^{*}\left(\boldsymbol{e} \cdot \nabla \eta^{k}\right)\right|_{z_{3}=0}\right\|_{W_{p}^{1-\frac{1}{p}}\left(\mathbb{R}^{2}\right)} \leq C\|\boldsymbol{e}\|_{W_{p}^{1}(K)} .
$$

Hence we obtain estimate (4.2).

Remark 4.2. The computations above were only formal as we had a priori no information about the existence of second derivatives of $\boldsymbol{e}$ or $\boldsymbol{E}$. However, recalling the uniqueness we may replace (4.6) by the following problem

$$
\begin{array}{lcc}
\Delta_{z} Z^{*} \boldsymbol{E}=\left(\Delta_{z}-\Delta_{x}\right) Z^{*} \widetilde{\boldsymbol{E}}+Z^{*} \boldsymbol{f}_{1} & \text { in } & \mathbb{R}_{+}^{3}, \\
Z^{*} \boldsymbol{E} \cdot \hat{\boldsymbol{e}}_{l}=0 & \text { on } & \mathbb{R}^{2}, \\
\left(Z^{*} \boldsymbol{E}\right)_{, z_{3}}^{(3)}=Z^{*}\left(\boldsymbol{e} \cdot \nabla \eta^{k}\right)-\left(\operatorname{div}_{x}-\operatorname{div}_{z}\right) Z^{*} \widetilde{\boldsymbol{E}} & \text { on } & \mathbb{R}^{2},
\end{array}
$$


and consider the mapping $T: \widetilde{\boldsymbol{E}} \mapsto \boldsymbol{E}$. Taking first $p=2$ due to estimates above it is not difficult to see that the mapping (for $\epsilon$ and $\lambda$ sufficiently small, the choice of $\epsilon$ depends on the transform $x \leftrightarrow z$ and $\lambda$ can be chosen arbitrarily) is a contraction in $W_{2}^{2}\left(\mathbb{R}_{+}^{3}\right)$; hence we prove the $W_{2(l o c)}^{2}\left(\bar{\Omega} ; \mathbb{R}^{3}\right)$ regularity for $\boldsymbol{e}$. Proceeding as for the interior case, i.e. first with $p=6$ and then any $p<\infty$, we finish the proof for arbitrary $p \in(1, \infty)$.

We need to remove the term $\|\boldsymbol{e}\|_{W_{p}^{1}(K)}$ from the right-hand side of (4.2). This is the aim of the following lemma.

Lemma 4.3. Let $\Omega$ be as above, $e \in L_{p(l o c)}\left(\Omega ; \mathbb{R}^{3}\right)$ and

$$
\Delta \boldsymbol{e} \in L_{p}\left(\Omega ; \mathbb{R}^{3}\right), \quad \boldsymbol{e} \cdot \vec{\tau}_{l}=0, \operatorname{div} \boldsymbol{e}=0 \text { on } \partial \Omega \quad \text { and } \quad \int_{\partial \Omega} \boldsymbol{e} \cdot \vec{n} \mathrm{~d} \sigma=0 .
$$

Then the following bound is valid

$$
\left\|\nabla^{2} \boldsymbol{e}\right\|_{L_{p}(\Omega)}+\left\|\left.\boldsymbol{e}\right|_{K}\right\|_{W_{p}^{1}(K)} \leq C\|\Delta \boldsymbol{e}\|_{L_{p}(\Omega)} .
$$

Proof. Let $K$ be a compact subset of $\bar{\Omega}$ fulfilling (2.3). First we want to show

$$
\|\boldsymbol{e}\|_{W_{p}^{1}(K)} \leq C\|\Delta \boldsymbol{e}\|_{L_{p}(\Omega)} .
$$

We show the above inequality by contradiction. If (4.18) is not true then there exists a sequence of $\left\{\boldsymbol{e}^{k}\right\}_{k \in \mathbb{N}}$ such that

Lemma 4.1 implies that

$$
\left\|e^{k}\right\|_{W_{p}^{1}(K)}>k\left\|\Delta e^{k}\right\|_{L_{p}(K)} \text { and }\left\|e^{k}\right\|_{W_{p}^{1}(K)}=1
$$

$$
\left\|\nabla^{2} \boldsymbol{e}\right\|_{L_{p}(\Omega)} \leq C\left(\|\Delta \boldsymbol{e}\|_{L_{p}(\Omega)}+\left\|\left.\boldsymbol{e}\right|_{K}\right\|_{W_{p}^{1}(K)}\right) .
$$

Hence we have

$$
\left\{\boldsymbol{e}^{k}\right\} \in W_{p}^{2}\left(K ; \mathbb{R}^{3}\right) \text { and }\left\|\boldsymbol{e}^{k}\right\|_{W_{p}^{2}(K)} \leq M \text { with }\left\|\Delta \boldsymbol{e}^{k}\right\|_{L_{p}(\Omega)} \rightarrow 0 \text { as } k \rightarrow \infty .
$$

By the Rellich theorem we find a subsequence

$$
\left.\left.\boldsymbol{e}^{k_{n}}\right|_{K} \rightarrow \boldsymbol{e}^{*}\right|_{K} \text { strongly in } W_{p}^{1}\left(K ; \mathbb{R}^{3}\right)
$$

On the other hand, $e^{*}$ fulfills

$$
\Delta \boldsymbol{e}^{*}=\mathbf{0} \text { in } \Omega, \quad \boldsymbol{e}^{*} \cdot \vec{\tau}_{l}=0, \quad \operatorname{div} \boldsymbol{e}^{*}=0 \text { on } \partial \Omega \text { with } \int_{\partial \Omega} \boldsymbol{e}^{*} \cdot \vec{n} \mathrm{~d} \sigma=0 .
$$

We get immediately $\operatorname{div} \boldsymbol{e}^{*}=0$ in $\Omega$, too. In particular we obtain $\Delta \boldsymbol{e}^{*}=-\operatorname{rot} \operatorname{rot} \boldsymbol{e}^{*}=\mathbf{0}$, then taking $\boldsymbol{v}^{*}=\operatorname{rot} \boldsymbol{e}^{*}$ we have the system

$$
\begin{array}{ll}
\operatorname{rot} \boldsymbol{v}^{*}=\mathbf{0} & \text { in } \Omega, \\
\operatorname{div} \boldsymbol{v}^{*}=0 & \text { in } \Omega, \\
\vec{n} \cdot \boldsymbol{v}^{*}=0 & \text { at } \partial \Omega .
\end{array}
$$

Since $\pi_{1}(\Omega)$ is trivial (the domain is simply connected), so by the Poincaré lemma (see [20]) rot $\boldsymbol{v}^{*}=\mathbf{0}$ yields the existence of a scalar function $p^{*}$ such that $\boldsymbol{v}^{*}=\nabla p^{*}$. It follows that system (4.22) is equivalent to the following Neumann problem

$$
\begin{array}{ll}
\Delta p^{*}=0 & \text { in } \Omega, \\
\vec{n} \cdot \nabla p^{*}=0 & \text { at } \partial \Omega .
\end{array}
$$

The maximum principle yields $\nabla p^{*}=0$, so $\boldsymbol{v}^{*} \equiv \mathbf{0}$ in $\Omega$. Then we get

$$
\begin{array}{rlrl}
\operatorname{rot} \boldsymbol{e}^{*}=\mathbf{0} \quad \text { in } & \Omega, \\
\operatorname{div} \boldsymbol{e}^{*}=0 \quad \text { in } & \Omega, \\
\vec{\tau}_{l} \cdot \boldsymbol{e}^{*}=0 & \text { at } \partial \Omega & l=1,2 .
\end{array}
$$


We see again that rot $\boldsymbol{e}^{*}=0$, so $\boldsymbol{e}^{*}=\nabla q^{*}$ which leads to the system

$$
\begin{aligned}
& \Delta q^{*}=0 \quad \text { in } \Omega, \\
& q^{*}=\text { const at } \partial \Omega,
\end{aligned} \text { and } \int_{\partial \Omega} \frac{\partial q^{*}}{\partial \vec{n}} \mathrm{~d} \sigma=0,
$$

since $\nabla q^{*} \cdot \vec{\tau}_{k}=0$ which gives $(4.25)_{2}$. The kernel of the Laplace operator for the Dirichlet problem in an exterior domain may possibly be nontrivial, but its behavior is determined by the fundamental solution. Hence one can easily deduce that the kernel function at $\partial \Omega$ achieves either its maximum or minimum. So the strong maximal principle says that the normal derivative on the boundary is strictly positive or negative, but this case is excluded by the integral constraint $\int_{\partial \Omega} \frac{\partial q^{*}}{\partial \vec{n}} \mathrm{~d} \sigma=0$. Whence $\boldsymbol{e}^{*} \equiv \mathbf{0}$.

On the other hand, $\boldsymbol{e}^{*}$ is a strong limit of $\boldsymbol{e}^{k}$ in $W_{p}^{1}(K)$, and by the choice of $\boldsymbol{e}^{k}$ we get $\left\|\boldsymbol{e}^{*}\right\|_{W_{p}^{1}(K)}=1$, what excludes $\boldsymbol{e}^{*}=\mathbf{0}$, leading to the contradiction. Estimate (4.18) is proved. Subsequently by (4.20) and the trace theorem we get (4.17).

Proof of Theorem 1.1 for the $L_{p}$ spaces. Taking results of Lemmas 4.1 and 4.3 we obtain the following priori estimate solutions to (2.1) with compactly supported sufficiently smooth right-hand side $\boldsymbol{f}$ :

$$
\left\|\nabla^{2} \boldsymbol{e}\right\|_{L_{p}(\Omega)}+\left\|\left.\boldsymbol{e}\right|_{K}\right\|_{W_{p}^{1}(K)} \leq C\|\boldsymbol{f}\|_{L_{p}(\Omega)} .
$$

Lemma 2.5 delivers a suitable approximation of $\boldsymbol{f}$. Then for a given $\epsilon>0$ we construct by Theorem 3.1 an approximative solution, which is a distributional one. Hence we are allowed to use the a priori estimate from Lemmas 4.1 and 4.3, i.e. we have

$$
\left\|\nabla^{2} \boldsymbol{e}_{\epsilon}\right\|_{L_{p}(\Omega)}+\left\|\left.\boldsymbol{e}_{\epsilon}\right|_{K}\right\|_{W_{p}^{1}(K)} \leq C\left\|\boldsymbol{f}_{\epsilon}\right\|_{L_{p}(\Omega)} .
$$

Passing with $\epsilon \rightarrow 0$ we get the desired solution to (2.1). Theorem 1.1 for the $L_{p}$ setting is finished. The uniqueness follows from the fact that zero function has a compact support.

Remark 4.4. We skip the considerations for Theorem 1.3, since they can be obtained by combining the technique presented above with corresponding estimates for the Besov spaces. The only point which is important to emphasize concerns the regularity of the boundary. In order to repeat considerations (4.11)-(4.12) we shall show that

$$
\|f g\|_{\dot{B}_{p, q}^{s}(\Omega)} \leq C\|f\|_{B_{r, q}^{s}(\Omega)}\|g\|_{B_{p, q}^{1+s}(\Omega)} .
$$

Recall that $r$ can be equal $p$ for $p>3$ (the dimension of $\Omega$ ) and $r>3$ as $p \leq 3$. We show (4.28) only for $s>0$ and $p \leq 3$ with $q=\infty$, the remaining cases are consequences of this case via interpolations or can be shown in a similar way. Thus

$$
\begin{aligned}
\|f g\|_{\dot{B}_{p, \infty}^{s}(\Omega)}= & \sup _{y \in \mathbb{R}^{3}}|y|^{-s}\|f g(\cdot-y)-f g\|_{L_{p}(\Omega)} \\
\leq & C\|f\|_{L_{r}(\Omega)} \sup _{y \in \mathbb{R}^{3}}|y|^{-s}\|g(\cdot-y)-g\|_{L_{m}(\Omega)} \\
& +C\|g\|_{L_{m}(\Omega)} \sup _{y \in \mathbb{R}^{3}}|y|^{-s}\|f(\cdot-y)-f\|_{L_{r}(\Omega)},
\end{aligned}
$$

where $\frac{1}{p}=\frac{1}{r}+\frac{1}{m}$, to use the imbedding theorem $B_{p, \infty}^{1+s} \subset B_{m, \infty}^{s}$.

The technique for the Besov spaces will be presented in detail during the proof of Theorem 1.4, i.e. for the critical case which is more difficult and also more interesting.

\section{The Limit Case $\dot{B}_{3,1}^{0}$}

As a limit case we have Theorem 1.4. We have to show that

$$
\left\|\nabla^{2} \boldsymbol{e}\right\|_{\dot{B}_{3,1}^{0}(\Omega)}+\|\boldsymbol{e}\|_{B_{3}^{1}(K)} \leq c\|\boldsymbol{f}\|_{\dot{B}_{3,1}^{0}(\Omega)} \text {, provided } \partial \Omega \in B_{3,1}^{2-1 / 3} .
$$


Note that for a "nice" right-hand side we have the existence of a solution in the space $W_{2(l o c)}^{1}\left(\Omega ; \mathbb{R}^{3}\right)$. First we have

Lemma 5.1. Let $\Omega$ be as in Theorem 1.4 and $\boldsymbol{e}$ be a sufficiently smooth vector field. Then the following estimate is valid

$$
\left\|\nabla^{2} \boldsymbol{e}\right\|_{\dot{B}_{3,1}^{0}(\Omega)} \leq C\left(\|\Delta \boldsymbol{e}\|_{\dot{B}_{3,1}^{0}(\Omega)}+\|\boldsymbol{e}\|_{B_{3,1}^{1}(K)}\right) .
$$

Proof. We follow the strategy introduced in the proof of Lemma 4.1, i.e. we use the localization technique. Let $\eta^{0}$ be as Lemma 4.1; denote $\boldsymbol{E}=\eta^{0} \boldsymbol{e}$, then for $\Delta \boldsymbol{e}=\boldsymbol{f}$ we immediately get

$$
\left\|\nabla^{2} \boldsymbol{E}\right\|_{\dot{B}_{3,1}^{0}\left(\mathbb{R}^{3}\right)} \leq C\left(\|\boldsymbol{f}\|_{\dot{B}_{3,1}^{0}\left(\mathbb{R}^{3}\right)}+\|\boldsymbol{e}\|_{B_{3,1}^{1}(K)}\right) .
$$

Next, take $x_{k} \in \partial \Omega$ and $Z^{x_{k}}$ with a parameter $\lambda_{k}=\lambda\left(x_{k}\right)>0$ - chosen for $x_{k}$, then we find

$$
\begin{array}{ll}
\Delta_{z} Z^{*} \boldsymbol{E}=\left(\Delta_{z}-\Delta_{x}\right) Z^{*} \boldsymbol{E}+Z^{*} \boldsymbol{f}_{1} & \text { in } \mathbb{R}_{+}^{3}, \\
Z^{*} \boldsymbol{E} \cdot \hat{\boldsymbol{e}}_{l}=0, l=1,2 & \text { on } \mathbb{R}^{2}, \\
\left(Z^{*} \boldsymbol{E}\right)_{z_{3}}^{(3)}=Z^{*}\left(\boldsymbol{e} \cdot \nabla \eta^{k}\right)+\left(\operatorname{div}_{z}-\operatorname{div}_{x}\right) Z^{*} \boldsymbol{E} & \text { on } \mathbb{R}^{2},
\end{array}
$$

where $\boldsymbol{f}_{1}$ is as in (4.5).

The a priori estimate for the half space shown in Lemma 2.6 yields

$$
\begin{aligned}
\left\|\nabla^{2} Z^{*} \boldsymbol{E}\right\|_{\dot{B}_{3,1}^{0}\left(\mathbb{R}_{+}^{3}\right)} \leq & C\left(\left\|Z^{*} \boldsymbol{f}_{\mathbf{1}}\right\|_{\dot{B}_{3,1}^{0}\left(\mathbb{R}_{+}^{3}\right)}+\left\|\left(\Delta_{x}-\Delta_{z}\right) Z^{*} \boldsymbol{E}\right\|_{\dot{B}_{3,1}^{0}(\Omega)}\right. \\
& \left.+\left\|\left(\operatorname{div}_{x}-\operatorname{div}_{z}\right) Z^{*} \boldsymbol{E}\right\|_{\dot{B}_{3,1}^{\frac{2}{3}}\left(\mathbb{R}^{2}\right)}+\left\|Z^{*}\left(\boldsymbol{e} \cdot \nabla e^{k}\right)\right\|_{\dot{B}_{3,1}^{\frac{2}{3}\left(\mathbb{R}^{2}\right)}}\right) .
\end{aligned}
$$

We estimate the r.h.s. of (4.3) in suitable norms. We get (cf. (4.9))

$$
\left\|\left(\Delta_{x}-\Delta_{z}\right) Z^{*} \boldsymbol{E}\right\|_{\dot{B}_{3,1}^{0}\left(\mathbb{R}_{+}^{3}\right)} \leq\left\|\left(\operatorname{div}_{z}-\operatorname{div}_{x}\right) \nabla_{x} Z^{*} \boldsymbol{E}\right\|_{\dot{B}_{3,1}^{0}\left(\mathbb{R}^{3}\right)}+\left\|\operatorname{div}_{x}\left(\nabla_{x}-\nabla_{z}\right) Z^{*} \boldsymbol{E}\right\|_{\dot{B}_{3,1}^{0}\left(\mathbb{R}^{3}\right)} .
$$

The main difficulty in estimation of the above term is, similarly as in the preceding section, the fact that we need to get a small parameter from the terms on the r.h.s. in (5.6). The standard paraproduct estimates would lead us to a result with a constant depending on norms of the boundary represented by maps $Z$ from Sect. 2, so we have to use the smallness of $\lambda$ and the property that $\operatorname{supp} Z^{*} \boldsymbol{E} \in B(0, \lambda) \cap \mathbb{R}_{+}^{3}$. Looking at the structure of terms in the r.h.s. of (5.6) we see that they reduce to the following form, note also that the considered regularity allows us to extend by symmetry these quantities on the whole space. Thus it is enough to consider

$$
\|B \nabla \boldsymbol{U}\|_{\dot{B}_{3,1}^{0}\left(\mathbb{R}^{3}\right)}+\|\nabla B \boldsymbol{U}\|_{\dot{B}_{3,1}^{0}\left(\mathbb{R}^{3}\right)},
$$

where $B \in \dot{B}_{3,1}^{1}\left(\mathbb{R}^{3}\right)$ corresponds to $d Z^{-1}(\cdot)-d Z^{-1}(0)$ and $\boldsymbol{U} \in \dot{B}_{3,1}^{1}\left(\mathbb{R}^{3} ; \mathbb{R}^{3}\right)$ corresponds to the gradient of $Z^{*} \boldsymbol{E}$, hence we additionally have $B(0)=0$ and $\operatorname{supp} \boldsymbol{U} \subset B_{\lambda}$.

Let us consider first the quantity $B \nabla \boldsymbol{U}$. For any given $\epsilon>0$ we find $B_{\epsilon}$ such that

$$
\left\|B-B_{\epsilon}\right\|_{\dot{B}_{3,1}^{1}\left(\mathbb{R}^{3}\right)} \leq \epsilon .
$$

Note now that $B_{\epsilon} \in C^{\infty} \subset W_{\infty}^{1}$ and $\nabla \boldsymbol{U}$ is supported in $B_{\lambda}$.

We apply Lemma 2.7. Taking $\epsilon>0$ sufficiently small we get

$$
\begin{aligned}
\|B \nabla \boldsymbol{U}\|_{\dot{B}_{3,1}^{0}\left(\mathbb{R}^{3}\right)} \leq & \left\|B_{\epsilon} \nabla \boldsymbol{U}\right\|_{\dot{B}_{3,1}^{0}\left(\mathbb{R}^{3}\right)}+\left\|\left(B-B_{\epsilon}\right) \nabla \boldsymbol{U}\right\|_{\dot{B}_{3,1}^{0}\left(\mathbb{R}^{3}\right)} \\
\leq & C\left(\left\|B_{\epsilon}\right\|_{L_{\infty}\left(\mathbb{R}^{3}\right)}+\lambda\left\|B_{\epsilon}\right\|_{\dot{W}_{\infty}^{1}\left(\mathbb{R}^{3}\right)}\|\nabla \boldsymbol{U}\|_{\dot{B}_{3,1}^{0}\left(\mathbb{R}^{3}\right)}\right. \\
& \left.+\left\|B-B_{\epsilon}\right\|_{\dot{B}_{3,1}^{1}\left(\mathbb{R}^{3}\right)}\|\nabla \boldsymbol{U}\|_{\dot{B}_{3,1}^{0}\left(\mathbb{R}^{3}\right)}\right) .
\end{aligned}
$$

First we determine the $\epsilon$ for a given $B$. Next, as $B(0)=0$,

$$
\left\|B_{\epsilon}\right\|_{L_{\infty}\left(\mathbb{R}^{3}\right)} \leq\|B\|_{L_{\infty}\left(\mathbb{R}^{3}\right)}+C\left\|\left(B-B_{\epsilon}\right)\right\|_{\dot{B}_{3,1}^{0}\left(\mathbb{R}^{3}\right)} \leq \omega(\lambda)+C \epsilon ;
$$

hence finally

$$
\|B \nabla \boldsymbol{U}\|_{\dot{B}_{3,1}^{0}\left(\mathbb{R}^{3}\right)} \leq C(\omega(\lambda)+\epsilon+\lambda \Xi[\epsilon, B])\|\nabla \boldsymbol{U}\|_{\dot{B}_{3,1}^{0}\left(\mathbb{R}^{3}\right)}
$$


The last quantity bounds $\left\|B_{\epsilon}\right\|_{W_{\infty}^{1}} \leq \Xi[\epsilon, B]$. Note that this quantity depends on the function $B$ and it can not be bounded by its norm. However, smallness of the parameter $\lambda$ makes it sufficiently small. Note that decreasing of $\lambda$ does not change features of $B$ which corresponds to the regularity of the boundary.

Now we return to the second term in (5.7). We use again the approximation of $B$

$$
\nabla B=\nabla B_{\epsilon}+\nabla\left(B-B_{\epsilon}\right) .
$$

Based on Lemma 2.8 and result from (5.9)-(5.11) we find that

$$
\begin{aligned}
\|\nabla B \boldsymbol{U}\|_{\dot{B}_{3,1}^{0}\left(\mathbb{R}^{3}\right)} & \leq\left\|\nabla B_{\epsilon} \boldsymbol{U}\right\|_{\dot{B}_{3,1}^{0}\left(\mathbb{R}^{3}\right)}+\left\|\nabla\left(B-B_{\epsilon}\right) \boldsymbol{U}\right\|_{\dot{B}_{3,1}^{0}\left(\mathbb{R}^{3}\right)} \\
& \leq C \Xi(\epsilon, B) \lambda\|\boldsymbol{U}\|_{\dot{B}_{3,1}^{1}\left(\mathbb{R}^{3}\right)}+C\left\|\nabla\left(B-B_{\epsilon}\right)\right\|_{\dot{B}_{3,1}^{0}\left(\mathbb{R}^{3}\right)}\|\boldsymbol{U}\|_{\dot{B}_{3,1}^{1}\left(\mathbb{R}^{3}\right)} \\
& \leq C(\epsilon+\lambda \Xi(\epsilon, B))\|\boldsymbol{U}\|_{\dot{B}_{3,1}^{1}\left(\mathbb{R}^{3}\right)} .
\end{aligned}
$$

As $\epsilon$ is small enough, taking $\lambda$ small we may control the magnitude of the term $\Xi(\epsilon, B)$.

Summing up

$$
\begin{aligned}
\left\|\nabla^{2} Z^{*} \boldsymbol{E}\right\|_{\dot{B}_{3,1}^{0}\left(\mathbb{R}^{3}\right)} \leq & M(\omega(\lambda)+\epsilon+\lambda \Xi(\epsilon, B))\left\|\nabla^{2} Z^{*} \boldsymbol{E}\right\|_{\dot{B}_{3,1}^{0}\left(\mathbb{R}^{3}\right)} \\
& +C(\lambda)\left(\|\boldsymbol{e}\|_{B_{3,1}^{1}(K)}+\|\boldsymbol{f}\|_{\dot{B}_{3,1}^{0}(\Omega)}\right) .
\end{aligned}
$$

Then

$$
\left\|\nabla^{2} Z^{*} \boldsymbol{E}\right\|_{\dot{B}_{3,1}^{0}\left(\mathbb{R}^{3}\right)} \leq C(\lambda)\left(\|\boldsymbol{e}\|_{B_{3,1}^{1}(K)}+\|\boldsymbol{f}\|_{\dot{B}_{3,1}^{0}(\Omega)}\right) .
$$

Note that for each $x_{k} \in \partial \Omega$ we may take a (strictly) positive $\lambda\left(x_{k}\right)$ and $B\left(x_{k}, \lambda_{k}\right)$. Indeed,

$$
\bigcup_{y \in \partial \Omega} B(y, \lambda(y) / 4) \supset \partial \Omega
$$

and by virtue of the compactness of the boundary $\partial \Omega$ we may find a finite subcovering, i.e. there exists $\left\{x_{k}\right\}_{k \in I}$ and $I=\left\{1, \ldots, N_{\partial \Omega}\right\}$ such that

$$
\bigcup_{k \in I} B\left(x_{k}, \lambda\left(x_{k}\right) / 4\right) \supset \partial \Omega
$$

Since $I$ is finite, the minimal $\lambda(x)$ is strictly positive, so the constants are uniformly bounded for this set. Summing up estimates (5.15) over the set $I$ we end up with (5.2).

Next, to show (5.1) we need

$$
\left\|\left.\boldsymbol{e}\right|_{K}\right\|_{B_{3,1}^{1}(K)} \leq C\|\Delta e\|_{\dot{B}_{3,1}^{0}(\Omega)} .
$$

The proof is exactly the same as for the $L_{p}$ case, see Lemma 4.3. It is enough to substitute $L_{p}(\Omega)$ by $\dot{B}_{3,1}^{0}(\Omega)$. Hence (5.1) is shown. This finishes the proof of Theorem 1.4.

Acknowledgments. P. B. Mucha has been partly supported by Polish Grant No. N201 547438 and by Foundation for Polish Science in fr. EU European Regional Development Funds (OPIE 2007-2013). The work of M. Pokorný has been supported by P201/11/1304 of Grant Agency of the Czech Republic.

Open Access. This article is distributed under the terms of the Creative Commons Attribution License which permits any use, distribution, and reproduction in any medium, provided the original author(s) and the source are credited.

\section{References}

[1] Amrouche, C., Seloula, N.: Lp-theory for vector potentials and Sobolev's inequalities for vector fields: application to the Stokes equations with pressure boundary conditions. Math. Models Methods Appl. Sci. 23(1), 37-92 (2013)

[2] Auchmuty, G., Alexander, J.C.: L2-well-posedness of 3D div-curl boundary value problems. Q. Appl. Math. 63(3), 479-508 (2005)

[3] Bennett, C., Sharpley, R.: Interpolation of Operators. Pure and Applied Mathematics, vol. 129. Academic Press, Inc., Boston (1988)

[4] Besov, O.V., I'lin, V.P., Nikolskij, S.M.: Integral Function Representation and Imbedding Theorem, Moscow (1975) 
[5] Bahouri, H., Chemin, J.Y., Danchin, R.: Fourier Analysis and Nonlinear Partial Differential Equations. Springer, Heidelberg (2011)

[6] Bolik, J., von Wahl, W.: Estimating $\nabla u$ in terms of $\operatorname{div} u, \operatorname{curl} u$, either $(v, u)$ or $v \times u$ and the topology. Math. Methods Appl. Sci. 20, 737-744 (1997)

[7] Danchin, R., Mucha, P.B.: A critical functional framework for the inhomogeneous Navier-Stokes equations. J. Funct. Anal. 256(3), 881-927 (2009)

[8] Danchin, R., Mucha, P.B.: The divergence equation in rough spaces. J. Math. Anal. Appl. 386(1), 9-31 (2012)

[9] Delcourte, S., Domelevo, K., Omnes, P.: A discrete duality finite volume approach to Hodge decomposition and div-curl problems on almost arbitrary two-dimensional meshes. SIAM J. Numer. Anal. 45(3), 1142-1174 (2007)

[10] Escher, J., Giga, Y., Ito, K.: On a limiting motion and self-intersections for the intermediate surface diffusion flow. J. Evol. Equ. 2(3), 349-364 (2002)

[11] Escher, J., Mucha, P.B.: The surface diffusion flow on rough phase spaces. Discrete Contin. Dyn. Syst. 26(2), 431-453 (2010)

[12] Escher, J., Simonett, G.: A center manifold analysis for the Mullins-Sekerka model. J. Differ. Equ. 143, 267-292 (1998)

[13] Friedrichs, K.O.: Differential forms on Riemannian manifolds. Commun. Pure Appl. Math. 8, 551-590 (1955)

[14] Galdi, G.P.: An Introduction to the Mathematical Theory of the Navier-Stokes Equations. Steady-State Problems, 2nd edn. Springer Monographs in Mathematics. Springer, New York (2011)

[15] Griffiths, D.J.: Introduction to Electrodynamics. Prentice Hall, Upper Saddle River (1999)

[16] Jackson, J.D.: Classical Electrodynamics, 3rd edn. Wiley, New York (1998)

[17] Kay, J.M.: An Introduction to Fluid Mechanics and Heat Transfer: With Applications in Chemical and Mechanical Process Engineering. Cambridge University Press, Cambridge (1963)

[18] Landau, L.D., Lifshitz, E.M.: Fluid Mechanics. Butterworth-Heinemann, UK (1987)

[19] Marcinkiewicz, J.: Sur les multiplicateurs des series de Fourier. Studia Math. 8, 78-91 (1939)

[20] Milnor, J.W., Stasheff, J.D.: Characteristic classes. Annals of Mathematics Studies, vol. 76. Princeton University Press, Princeton (1974)

[21] Mucha, P.B.: On weak solutions to the Stefan problem with Gibbs-Thomson correction. Differ. Integr. Equ. 20, $769-792(2007)$

[22] Mucha, P.B.: On the Stefan problem with surface tension in the Lp framework. Adv. Differ. Equ. 10, 861-900 (2005)

[23] Mucha, P.B., Zajaczkowski, W.: On local existence of solutions of the free boundary problem for an incompressible viscous self-gravitating fluid motion. Appl. Math. (Warsaw) 27, 319-333 (2000)

[24] Nicolaides, R.A., Wu, X.: Covolume, solutions of three-dimensional div-curl equations. SIAM J. Numer. Anal. 34(6), 2195-2203 (1997)

[25] Runst, T., Sickel, W.: Sobolev spaces of fractional order, Nemytskij operators, and nonlinear partial differential equations. de Gruyter Ser. Nonlinear Anal., Berlin (1996)

[26] Solonnikov, V.A.: Overdetermined elliptic boundary value problems. Zap. Nauch. Sem. LOMI 21, 112-158 (1971)

[27] Solonnikov, V.A.: On the nonstationary motion of an isolated volume of a viscous incompressible fluid. Izv. Akad. Nauk SSSR 51, 1065-1087 (1987)

[28] Tomoro, A.: On smoothing effect for higher order curvature flow equations. Adv. Math. Sci. Appl. 20(2), 483-509 (2010)

[29] Triebel, H.: Interpolation Theory, Function Spaces, Differential Operators. North-Holland Math. Library, vol. 18. NorthHolland Publishing Co., Amsterdam (1978)

[30] von Wahl, W.: Estimating $\nabla u$ by div $u$ and curl $u$. Math. Methods Appl. Sci. 15, 123-143 (1992)

[31] Zajaczkowski, W.: Existence and regularity of some elliptic systems in domains with edges. Dissertationes Math. (Rozprawy Mat.) 274 (1989)

[32] Zajaczkowski, W.: Global Special Regular Solutions to the Navier-Stokes Equations in a Cylindrical Domain Under Boundary Slip Conditions. Gakuto International Series, Mathematical Sciences and Applications, vol. 21. Gakkotosho, Tokyo (2004)

Piotr B. Mucha

Institute of Applied Mathematics and Mechanics

University of Warsaw

ul. Banacha 2, 02-097 Warszawa, Poland

e-mail: p.mucha@mimuw.edu.pl

(accepted: April 21, 2014; published online: August 7, 2014)
Milan Pokorný

Charles University in Prague

Faculty of Mathematics and Physics

Mathematical Institute of Charles University

Sokolovská 83, 18675 Praha, Czech Republic

e-mail: pokorny@karlin.mff.cuni.cz 